\title{
CONSISTENT RECALIBRATION OF YIELD CURVE MODELS
}

\author{
PHILIPP HARMS, DAVID STEFANOVITS, JOSEF TEICHMANN, AND MARIO V. WÜTHRICH
}

\begin{abstract}
The analytical tractability of affine (short rate) models, such as the Vasiček and the Cox-Ingersoll-Ross models, has made them a popular choice for modelling the dynamics of interest rates. However, in order to account properly for the dynamics of real data, these models need to exhibit time-dependent or even stochastic parameters. This in turn breaks their tractability, and modelling and simulating becomes an arduous task. We introduce a new class of Heath-Jarrow-Morton (HJM) models that both fit the dynamics of real market data and remain tractable. We call these models consistent recalibration (CRC) models. These CRC models appear as limits of concatenations of forward rate increments, each belonging to a Hull-White extended affine factor model with possibly different parameters. That is, we construct HJM models from "tangent" affine models. We develop a theory for a continuous path version of such models and discuss their numerical implementations within the Vasiček and Cox-Ingersoll-Ross frameworks.
\end{abstract}

\section{INTRODUCTION}

1.1. Principles of yield curve modelling. Modelling the stochastic evolution of yield curves is an important task in risk management, forecasting, decision making, pricing and hedging. We emphasise here three principles of yield curve modelling (or any other traded instrument in finance): we certainly require that all models for traded assets' prices are free of arbitrage; therefore we do not state this as a principal requirement.

- Robust calibration: the model is selected simultaneously from time series and prevailing market prices, as explained in [12. Model parameters which are invariant under equivalent measure changes should be estimated by a statistical procedure from time series data. The remaining parameters are calibrated by solving an inverse problem with respect to the prevailing market prices. All model parameters should be constant during the life time of the model; only state variables may change.

- Consistency: an interest rate model is called consistent if the stochastic process of yield curves does not leave a pre-specified set $\mathcal{I}$ of possible market observables (in [16 the set $\mathcal{I}$ is assumed to be a finite dimensional sub-manifold of curves corresponding to a curve fitting method). Here, we add the following requirement: the yield curve process should be able to reach any neighbourhood of any yield curve in $\mathcal{I}$ with positive probability because any newly arriving market configuration is a possible model state. Consequently, the model can be recalibrated to a new market configuration without losing consistency to the model choice with old parameters; we say that the model satisfies the consistent recalibration property.

Date: September 2016.

2010 Mathematics Subject Classification. 91G30, 60J25, 60J60 .

Supported in part by SNF grant 149879.

We gratefully acknowledge support by ETH Foundation. 
- Analytic tractability: relevant quantities of a model can be calculated quickly and accurately. In particular, one should be able to simulate state variable increments efficiently. This can be a delicate problem in the presence of boundary conditions.

We briefly comment on some of these principles.

- By a model for the term structure of interest rates, we understand a fully specified stochastic process taking values in the pre-specified set of yield curves $\mathcal{I}$. We shall always consider a parametrised class of models consisting of one fully specified model for each initial state in $\mathcal{I}$ and each parameter value.

- In practice, interest rate models are recalibrated on a regular basis (e.g. daily) to market data. Suppose that the consistent recalibration property does not hold for today's model. Then tomorrow's market yield curve might lie outside of the set of possible realisations of today's model. If this happens, then tomorrow's recalibration necessarily implicates a rejection of today's model. On the other hand, no inconsistencies occur if recalibration is an update of the state variables of the model and does not involve a change of model parameters.

- The robust calibration principle separates the easier task of estimating volatilities from the more difficult task of estimating drifts. Moreover, it tells us exactly which parameters may be estimated from time series (namely those which are invariant under equivalent changes of measure). In 22 we use our results to model and filter the market price of risk.

1.2. Consistent recalibration models. The goal of this work is to present a new model class for yield curve evolutions satisfying all three principal requirements with respect to sets $\mathcal{I}$ which are sufficiently large to be of practical use (think of open subsets of a Hilbert space of curves). Mathematically speaking, we look for yield curve models with full or large support, which are in addition analytically tractable.

Often the full support property does not accord with analytic tractability beyond elliptic models, which are too restrictive in infinite dimension. We illustrate this with an example. Consider the Hull-White extended Cox-Ingersoll Ross (CIR) model. In this model the short rate is given by the SDE

$$
d r(t)=(\theta(t)+\beta r(t)) d t+\sqrt{\alpha r(t)} d W(t),
$$

where $\theta(t) \geq 0$ determines the time-dependent level of mean reversion, $\beta<0$ the speed of mean reversion, and $\alpha>0$ the level of volatility.

The model can be calibrated to any initial yield curve from a large subset $\mathcal{I}$ of curves by choosing an appropriate Hull-White extension $\theta$. For any fixed initial yield curve, the distribution of yield curves at some future $t>0$ is concentrated on a one-dimensional affine subspace of curves. Therefore, market observations are generally not in the support of the model, and the consistent recalibration property does not hold with respect to $\mathcal{I}$. Moreover, the low dimensionality of the model is apparent at the level of realised covariations of yields for different times to maturity: the matrix of covariations has rank one, which is in stark contrast to observations from the market (see Figure 7.13). Finally, calibrated model parameters vary significantly over time as shown in Figures 7.4 and 7.6 . which contradicts the requirement of robust calibration.

As a remedy, one could make some model parameters stochastic and include them as state variables. For example, one could make $\alpha=\alpha_{y}$ and $\beta=\beta_{y}$ depend on a parameter 
$y$ and write dynamics of the form

$$
\begin{aligned}
d r(t) & =\left(\theta(t)+\beta_{Y(t)} r(t)\right) d t+\sqrt{\alpha_{Y(t)} r(t)} d W(t), \\
d Y(t) & =\mu(Y(t)) d t+\sigma(Y(t)) d \widetilde{W}(t) .
\end{aligned}
$$

Unfortunately, this usually breaks the analytic tractability of the model in the sense that zero-coupon bond prices cannot be calculated anymore analytically.

The key idea of consistent recalibration (CRC) models is to lift the short rate model to a HJM model and to introduce stochastic parameters on that level. Let $h(t)$ denote the forward rate curve at time $t$ in Musiela parametrisation (i.e., as a function of time to maturity). Then CRC models are defined by the joint dynamics

$$
\begin{aligned}
d h(t) & =\left(\mathcal{A} h(t)+\mu_{Y(t)}^{\mathrm{HJM}}(r(t))\right) d t+\sigma_{Y(t)}^{\mathrm{HJM}}(r(t)) d W(t), \\
d Y(t) & =\mu(Y(t)) d t+\sigma(Y(t)) d \widetilde{W}(t),
\end{aligned}
$$

where $r(t)=h(t)(0), \mathcal{A}$ is the generator of the shift semigroup, and $\mu_{y}^{\mathrm{HJM}}, \sigma_{y}^{\mathrm{HJM}}$ are the HJM drift and volatility of the short rate model with constant parameter $y$. This model, and more generally the class of CRC models, has the following properties:

- It is a full-fledged HJM model providing the benefits of robust calibration. Indeed, all model parameters can be estimated from realised covariations of yields rather than calibrated by solving high-dimensional inverse problems. Thus, the parameters are estimated from yield curve dynamics instead of calibrated to static yield curves, while an exact match to the current yield curve is guaranteed by the Hull-White extension $\theta$.

- In the language of term structure equations, see 29, 9, 24, CRC models are tangent to affine factor models. This means that they are (limits of) concatenations of affine factor models. The construction is illustrated in Figure 4.2. The concatenated models are allowed to have distinct static parameters. Making the parameters stochastic (in an independent or dependent way) will lead generically towards consistent recalibration, since the conditions for finite-dimensional realisations are not fulfilled anymore. The consistent recalibration property is also reflected in the much higher ranks of the covariation matrices of yields with different times to maturity. Indeed, our empirical analysis shows that they are closer to those observed in the market than in the corresponding models without CRC extension (see Figure 7.13).

- Despite all this flexibility, the model remains analytically tractable: zero-coupon bond prices are state variables, and state variable increments can be simulated efficiently because they look infinitesimally like Hull-White extended affine processes. This means that the Fourier transform of the infinitesimal increments is known and that all sampling techniques of affine processes apply. In particular, efficient high-order positivity-preserving simulation schemes for the CIR process such as 11 can be used. No similar schemes are available for general HJM equations with non-Lipschitz vector fields. In our numerical implementation, we achieve first-order convergence of the splitting scheme. We also give a theoretical proof of first-order convergence in the Vasiček case.

These properties are important in risk management and in the current regulatory framework 31, where one needs tractable and realistic (non-Gaussian) models of long-term returns on bond portfolios. Moreover, the CRC approach allows one to easily implement 
stress tests for risk management purposes by selecting a suitable model for the parameter process. First evidence of improved fits is provided in 22 .

The principle behind CRC models applies also to the modelling of more general term structure dynamics. For example, it could be applied to multi-curve interest rate models and to models of the term structure of option prices [29].

1.3. Organisation of the paper. In Section 2 we discuss relations to other yield curve modelling approaches. In Section 3, we introduce Hull-White extended affine short rate models, which are the building blocks of CRC models introduced in Section 4 . In Sections 5 and 6, the one-factor Vasiček and CIR case is developed in full detail. In Section 7, our numerical implementation and some empirical results are presented.

\section{Relations to OtheR YIELD CURVE MOdeLS}

Several in part overlapping approaches to yield curve modelling have been developed. The models can be roughly categorised as factor, HJM, principal component analysis (PCA), and filtered historical simulation models. We briefly analyse these models with respect to our requirements and compare them to the new class of CRC models.

2.1. Factor models. Factor models are based on a factor process, which usually describes certain market factors, from which - by means of basic principles - the entire yield curve can be derived (see [17 for an overview). Let $X=(X(t))_{t \geq 0}$ be a factor Markov process acting on a finite dimensional state space and depending on a parameter vector $y$, and let $B:=g(X)$ be the bank account process, for some positive functional $g$. Then one obtains - with respect to the pricing measure $\mathbb{P}$ - the relation

$$
P(t, T)=\mathbb{E}\left[\frac{B(t)}{B(T)} \mid \mathcal{F}(t)\right]=G(t, T, X(t)),
$$

for some function $G$ also depending on the parameter vector $y$.

Market data arrive in the form of daily yield curves. By means of calibration the initial state $x_{0}$ and a parameter vector $y_{0}$ are chosen to explain today's market data. By choosing the parameter vector rich enough, one receives good fits to today's market data. Apparently a recalibration at time $t=1$ can (and will) lead to another state $x_{1}$ and another parameter vector $y_{1}$. As states may vary stochastically, the change of $x_{0}$ to $x_{1}$ is in principle not a problem, but the change of parameter vector is. This means that one has to decide at time $t=1$ whether to continue with the model specified by $y_{0}$ or whether to switch to the model specified by $y_{1}$. This problem can be alleviated to some extent by using a combination of filtering and calibration techniques to stabilise the choice of $y$, as described in, e.g., [19, 4]. Nevertheless, robust calibration remains an unresolved issue.

The consistent recalibration property does not hold unless the set $\mathcal{I}$ is very small. (If $\mathcal{I}$ is a sub-manifold, its dimension cannot be larger than that of the factor process.) However, on the positive side, factor models are often analytically tractable, for instance, within the affine class (see e.g. 17, 15]).

2.2. HJM-models. Markovian HJM models are an extreme version of factor models: the yield curve itself is taken as state variable (possibly together with some hidden state variables). Calibration to daily arriving yield curves is now a matter of statistical estimation from the time series of market data. An appropriate parametrisation of instantaneous co-variance, jump structure, and drifts will lead to a statistical inference problem, an infinite dimensional one though. Hence, the paradigm of robust calibration, including 
the requirement of calibration through estimation, is fulfilled in the optimal sense. If the process acting on yield curves is "irreducible", i.e., every neighbourhood of a state can be reached with positive probability, then even consistent recalibration is possible (see [5]). However, one usually encounters a severe lack of analytic tractability within this model class. Euler and higher order schemes (often) require strong assumptions on the vector fields (c.f. 14]). Usually no exact or high-order simulations of infinitesimal increments are at hand in contrast to CRC models, where this is often the case.

2.3. PCA- or local PCA models. Principal component analysis (PCA) or local PCA considers yield curves as outcomes of a statistical model, which is estimated by standard PCA techniques (see e.g. 26, 6, 11]). When the statistical model is too simplistic, often arbitrage enters the field, which is an undesirable feature. A more refined version is actually equivalent to a HJM model with constant vector fields (as e.g. in [23]). Here preserving floored interest rates, which is desired in some situations, is not possible. PCA inspired models, correctly implemented, allow for robust calibration and consistent recalibration, but are usually not very tractable from an analytic point of view.

2.4. Filtered (historical) simulation. Historical simulation is a standard industry technique to simulate distributions of yield curves by considering the relative returns as independent samples of an unknown distribution, see [19, 4]. Certainly this assumption can lead to difficulties with the absence of arbitrage, but this can be solved as in 28,32 . The most important problem is the state-independence of the distribution. Again also (filtered) historical simulation can be embedded into the realm of HJM models. These models then allow for robust calibration and consistent recalibration, but are usually not very tractable from an analytic point of view.

\section{Hull-White extended AFFine Short RAte Models}

3.1. Overview. We set the stage for CRC models by describing Hull-White extended affine short rate models, focusing first on the correspondence between Hull-White extensions and initial forward rate curves. The one-dimensional short rate model of the introduction is replaced by more general multi-dimensional factor models for the short rate. The parameter $y$, which becomes stochastic in the CRC setting, is kept constant and fixed for the moment.

3.2. Setup and notation. $\left(\Omega, \mathcal{F},(\mathcal{F}(t))_{t \geq 0}, \mathbb{P}\right)$ is a filtered probability space. The filtration satisfies the usual conditions. The measure $\mathbb{P}$ plays the role of a risk-neutral measure. All processes are defined on $\Omega$, adapted to $(\mathcal{F}(t))_{t \geq 0}$, and càdlàg. $W=(W(t))_{t \geq 0}$ is a $d$-dimensional $(\mathcal{F}(t))_{t \geq 0}$-Brownian motion.

The short rate process $r=(r(t))_{t \geq 0}$ is determined by a factor process $X=(X(t))_{t \geq 0}$ with values in a state space $\mathbb{X}$. The evolution of the factor process depends on a parameter process $Y=(Y(t))_{t \geq 0}$ with values in a space $\mathbb{Y}$. In all of Section 3, the parameter process $Y(t) \equiv y$ is assumed to be constant and fixed, whereas it is allowed to vary in Section 4 below.

The spaces $\mathbb{X}$ and $\mathbb{Y}$ are both subsets of some finite dimensional vector space. $\mathbb{X}$ is, up to permutation of coordinates, of the canonical form $\mathbb{R}_{+}^{d_{1}} \times \mathbb{R}^{d_{2}}$ with $d_{1}+d_{2}=d \geq 1$. The canonical basis vectors in $\mathbb{R}^{d}$ are denoted by $e_{1}, \ldots, e_{d}$, and $\langle\cdot, \cdot\rangle$ denotes the Euclidean scalar product. Of course we could consider more general affine processes here, which take values, e.g., in products of cones of positive-semidefinite matrices and real lines like Wishart-Heston models. 
For each $(x, y) \in \mathbb{X} \times \mathbb{Y}$, there is a symmetric positive semidefinite matrix $A_{y}(x) \in \mathbb{R}^{d \times d}$ and a vector $B_{y}(x) \in \mathbb{R}^{d}$, determining the volatility and the drift of $X$. The expressions $A_{y}(x)$ and $B_{y}(x)$ are affine in $x$, i.e.,

$$
A_{y}(x)=a_{y}+\sum_{i=1}^{d} \alpha_{y}^{i} x^{i}, \quad B_{y}(x)=b_{y}+\sum_{i=1}^{d} \beta_{y}^{i} x^{i}, \quad \text { for all }(x, y) \in \mathbb{X} \times \mathbb{Y},
$$

for symmetric positive semidefinite matrices $a_{y}, \alpha_{y}^{1}, \ldots, \alpha_{y}^{d} \in \mathbb{R}^{d \times d}$ and $b_{y}, \beta_{y}^{1}, \ldots, \beta_{y}^{d} \in \mathbb{R}^{d}$. We denote by $\sqrt{A_{y}(x)}$ the symmetric positive semidefinite square root of $A_{y}(x)$. Note: other choices of square roots are possible, and Assumption 3.1 below does not depend on the choice of square root by [30, Chapter V.19-20]. Moreover, a function $\theta \in C\left(\mathbb{R}_{+}\right)$is given, which is used to make the drift of $X$ time-inhomogeneous.

3.3. Factor process and short rate. The factor process $X$ is a continuous, $\mathbb{X}$-valued solution of the SDE

$$
d X(t)=\sqrt{A_{y}(X(t))} d W(t)+\left(\theta(t) e_{1}+B_{y}(X(t))\right) d t
$$

with initial condition $X(0)=x \in \mathbb{X}$. The short rate is given by

$$
r(t)=\ell+\langle\lambda, X(t)\rangle, \quad \text { for all } t \geq 0,
$$

for some fixed $\ell \in \mathbb{R}$ and $\lambda \in \mathbb{R}^{d}$ satisfying $\left\langle\lambda, e_{1}\right\rangle \neq 0$.

Assumption 3.1. It is assumed that SDE (3.1) has a unique continuous, $\mathbb{X}$-valued solution $X$, for each initial condition $X(s)=x$, where $(s, x) \in \mathbb{R}_{+} \times \mathbb{X}$. In this case, the parameters $(y, \theta)$ are called admissible. Moreover, it is assumed that $X$ satisfies the moment condition

$$
\mathbb{E}\left[e^{-\int_{0}^{t}(\ell+\langle\lambda, X(s)\rangle) d s}\right]<\infty, \quad \text { for all } t \geq 0 .
$$

3.4. Exponential moments and Riccati equations. The process $X$, or rather the family of processes obtained by varying the initial conditions in SDE (3.1), is timeinhomogeneous affine. All coefficients in SDE (3.1) are independent of time, except for the drift $\theta$; we call $X$ Hull-White extended affine and $\theta$ its Hull-White extension. This we are going to highlight in detail below. Our main reference for time-inhomogeneous affine processes is [18].

Functions $\left(\Phi_{y}, \Psi_{y}\right) \in C^{\infty}\left(\mathbb{R}_{+}\right) \times C^{\infty}\left(\mathbb{R}_{+} ; \mathbb{R}^{d}\right)$ are called solutions of the Riccati equations (with parameter $y \in \mathbb{Y}$ ) if

$$
\begin{aligned}
& \Phi_{y}^{\prime}=F_{y} \circ \Psi_{y}, \\
& \Phi_{y}(0)=0, \\
& \Psi_{y}^{\prime}=R_{y} \circ \Psi_{y}-\lambda, \\
& \Psi_{y}(0)=0
\end{aligned}
$$

holds, where $\left(F_{y}, R_{y}\right) \in C\left(\mathbb{R}^{d}\right) \times C\left(\mathbb{R}^{d} ; \mathbb{R}^{d}\right)$ are given by

$$
F_{y}(u)=\frac{1}{2}\left\langle u, a_{y} u\right\rangle+\left\langle u, b_{y}\right\rangle, \quad R_{y}^{i}(u)=\frac{1}{2}\left\langle u, \alpha_{y}^{i} u\right\rangle+\left\langle u, \beta_{y}^{i}\right\rangle,
$$

for all $u \in \mathbb{R}^{d}$ and $i \in\{1, \ldots, d\}$.

Lemma 3.2. Let $X$ be a solution of $S D E$ (3.1) for some admissible parameters $(y, \theta)$. Then $X$ satisfies moment condition (3.3) if and only if there exists a solution $\left(\Phi_{y}, \Psi_{y}\right)$ of the Riccati equations (3.4) for all times $t \geq 0$. Moreover, if there exists a solution, even a local one, of the Riccati equations, it is unique. 
Note that the Riccati equations (3.4) only depend on $y$, and not on the choice of the Hull-White extension $\theta$.

Proof. Let $Z$ be the $\mathbb{X}^{2}$-valued process $Z(t)=\left(Z_{1}(t), Z_{2}(t)\right)=\left(X(t), \int_{0}^{t} X(s) d s\right)$. Then $Z$ is an Itō diffusion whose drift and volatility at time $t \geq 0$ are given by

$$
\left(\theta(t) e_{1}+B_{y}\left(Z_{1}(t)\right), Z_{1}(t)\right) \in \mathbb{R}^{2 d}, \quad\left(\begin{array}{cc}
A_{y}\left(Z_{1}(t)\right) & 0 \\
0 & 0
\end{array}\right) \in \mathbb{R}^{2 d \times 2 d},
$$

respectively. Clearly, these expressions are affine in $Z(t)$. Moreover, the time-inhomogeneity $\theta(t) e_{1}$ is (by definition) continuous in $t$. Therefore, the process $Z$, or rather the family of processes obtained by varying the initial condition of $Z$, is strongly regular affine, see 18 , Theorem 2.14]. For each $\left(t, u_{1}, u_{2}\right) \in \mathbb{R}_{+} \times \mathbb{R}^{d} \times \mathbb{R}^{d}$, the functional characteristics of $Z$ are given by

$$
\begin{aligned}
& F\left(t, u_{1}, u_{2}\right)=\theta(t)\left\langle u_{1}, e_{1}\right\rangle+F_{y}\left(u_{1}\right) \in \mathbb{R}, \\
& R\left(t, u_{1}, u_{2}\right)=\left(R_{y}\left(u_{1}\right)+u_{2}, 0\right) \in \mathbb{R}^{d} \times \mathbb{R}^{d} .
\end{aligned}
$$

Moment condition (3.3), expressed in terms of $Z$, reads as follows:

$$
\mathbb{E}\left[e^{-\left\langle\lambda, Z_{2}(T)\right\rangle}\right]<\infty, \quad \text { for all } T \geq 0
$$

By [21], the moment condition is equivalent to the existence of a solution $\left(\phi, \psi_{1}, \psi_{2}\right)$ of the following Riccati system associated to $Z$ :

$$
\begin{aligned}
-\partial_{t} \phi(t, T) & =\theta(t)\left\langle\psi_{1}(t, T), e_{1}\right\rangle+F_{y}\left(\psi_{1}(t, T)\right), & \phi(T, T) & =0, \\
-\partial_{t} \psi_{1}(t, T) & =R_{y}\left(\psi_{1}(t, T)\right)+\psi_{2}(t, T), & \psi_{1}(T, T) & =0, \\
-\partial_{t} \psi_{2}(t, T) & =0, & \psi_{2}(T, T) & =-\lambda .
\end{aligned}
$$

Equivalently, the relations $\psi_{2}(t, T)=-\lambda$ and

$$
\phi(t, T)=\int_{t}^{T} \theta(s)\left\langle\Psi_{y}(T-s), e_{1}\right\rangle d s+\Phi_{y}(T-t), \quad \psi_{1}(t, T)=\Psi_{y}(T-t)
$$

hold identically, where $\left(\Phi_{y}, \Psi_{y}\right)$ is a solution of the Riccati equations 3.4. Uniqueness holds for these equations because the vector fields are locally Lipschitz.

3.5. Bond prices and forward rates. By no-arbitrage arguments zero-coupon bond prices in the short rate model of Section 3.3 are given by

$$
P(t, T)=\mathbb{E}\left[e^{-\int_{t}^{T} r(s) d s} \mid \mathcal{F}(t)\right]=\mathbb{E}\left[e^{-\int_{t}^{T}(\ell+\langle\lambda, X(s)\rangle) d s} \mid \mathcal{F}(t)\right], \quad T \geq t \geq 0 .
$$

For essentials on short rate models we refer to 17 , Chapter 5]. We define the (instantaneous) forward rates by

$$
h(t, \tau)=h(t)(\tau)=-\partial_{\tau} \log (P(t, t+\tau)), \quad t, \tau \geq 0 .
$$

The parametrisation of the forward rate as a function of $t$ and $\tau$ is called Musiela parametrisation. It is particularly useful in this paper since $(h(t))_{t \geq 0}$ will be interpreted as a stochastic process taking values in a suitable space of functions on $\mathbb{R}_{+}$.

By the affine nature of the factor process $X$, zero-coupon bond prices and forward rates can be obtained by solving the Riccati system of ODE's 3.4. 
Theorem 3.3 (Zero-coupon bond price and forward rate). Let X satisfy moment condition (3.3) and let $\left(\Phi_{y}, \Psi_{y}\right)$ be the unique solution of the Riccati equations with parameter $y$, given by Lemma 3.2. Then the bond prices in the short rate model (3.1)-(3.2) satisfy

$$
\log (P(t, T))=-\ell(T-t)+\int_{t}^{T} \theta(s)\left\langle\Psi_{y}(T-s), e_{1}\right\rangle d s+\Phi_{y}(T-t)+\left\langle\Psi_{y}(T-t), X(t)\right\rangle,
$$

for all $T \geq t \geq 0$, and the forward rates are given by

$$
h(t, \tau)=\ell-\int_{0}^{\tau} \theta(t+s)\left\langle\Psi_{y}^{\prime}(\tau-s), e_{1}\right\rangle d s-\Phi_{y}^{\prime}(\tau)-\left\langle\Psi_{y}^{\prime}(\tau), X(t)\right\rangle
$$

for all $t, \tau \geq 0$.

Proof. We borrow from the proof of Lemma 3.2, where moment condition (3.3) was shown to be equivalent to the existence of solutions $\left(\phi, \psi_{1}, \psi_{2}\right)$ of the Riccati system associated to the process $Z=\left(X, \int X\right)$. Moreover, $\left(\phi, \psi_{1}, \psi_{2}\right)$ are closely related to the solutions $\left(\Phi_{y}, \Psi_{y}\right)$ of Riccati system (3.4). By the main theorem in 21] and its conditional version, the affine transform formula

$$
\mathbb{E}\left[e^{-\left\langle\lambda, Z_{2}(T)\right\rangle} \mid \mathcal{F}(t)\right]=e^{\phi(t, T)+\left\langle\psi_{1}(t, T), Z_{1}(t)\right\rangle+\left\langle\psi_{2}(t, T), Z_{2}(t)\right\rangle}, \quad \text { for all } T \geq t \geq 0,
$$

holds. A direct calculation shows this formula to be equivalent to formula (3.5 for bond prices. Formula 3.6 for forward rates is obtained by taking the logarithm and differentiating with respect to $\tau$.

3.6. Heath-Jarrow-Morton equation. The evolution of forward rate curves is described by the HJM equation. For each $(x, y) \in \mathbb{X} \times \mathbb{Y}$, let $\mu_{y}^{\mathrm{HJM}}(x)$ and $\sigma_{y}^{\mathrm{HJM}}(x)$ be given by

$$
\mu_{y}^{\mathrm{HJM}}(x)=\left\langle\Psi_{y}, A_{y}(x) \Psi_{y}^{\prime}\right\rangle \in C^{\infty}\left(\mathbb{R}_{+}\right), \quad \sigma_{y}^{\mathrm{HJM}}(x)=-\sqrt{A_{y}(x)} \Psi_{y}^{\prime} \in C^{\infty}\left(\mathbb{R}_{+} ; \mathbb{R}^{d}\right) .
$$

Note that the familiar HJM drift condition holds:

$$
\mu_{y}^{\mathrm{HJM}}(x)(\tau)=\left\langle\sigma_{y}^{\mathrm{HJM}}(x)(\tau), \int_{0}^{\tau} \sigma_{y}^{\mathrm{HJM}}(x)(s) d s\right\rangle, \quad \text { for all } \tau \geq 0 .
$$

Let $\mathbb{H}$ be a Hilbert space, destined to contain the forward rate curves of the model. By abuse of notation the symbol $h$ is used interchangeably to denote an element of $\mathbb{H}$ and the forward rate process.

Assumption 3.4. $\mathbb{H}$ is a Hilbert space with the following properties:

(i) $\mathbb{H} \subset C\left(\mathbb{R}_{+}\right)$and the evaluation map eval $\tau_{\tau}: h \mapsto h(\tau)$ is continuous on $\mathbb{H}$, for each $\tau \in \mathbb{R}_{+}$

(ii) for each $(x, y, z) \in \mathbb{X} \times \mathbb{Y} \times \mathbb{R}^{d}, \mu_{y}^{\mathrm{HJM}}(x)$ and $\left\langle\sigma_{y}^{\mathrm{HJM}}(x), z\right\rangle$ are elements of $\mathbb{H}$;

(iii) the right shifts $(\mathcal{S}(t))_{t \geq 0}$ mapping $h$ to $h(t+\cdot)$ define a strongly continuous semigroup on $\mathbb{H}$ with infinitesimal generator $\mathcal{A}$.

Hilbert spaces of forward rate curves which comply with the requirements of Assumption 3.4 are constructed in 16. Sections 5, 7.4.1 and 7.4.2] for the Vasiček and CIR models. In the domain $\mathcal{D}(\mathcal{A}) \subset \mathbb{H} \cap C^{1}\left(\mathbb{R}_{+}\right)$(c.f. [16, Lemma 4.2.2]) of the infinitesimal generator $\mathcal{A}$ we can characterise the process $(h, X)$ as follows. 
Theorem 3.5 (HJM equation). Let $(h, X)$ be given by Theorem 3.3 and assume that $h(t) \in \mathcal{D}(\mathcal{A})$, for each $t \geq 0$. Then the process $(h, X)$ is a strong solution of the following $S P D E$ on $\mathbb{H} \times \mathbb{X}$ :

$$
\begin{aligned}
d h(t) & =\left(\mathcal{A} h(t)+\mu_{y}^{\mathrm{HJM}}(X(t))\right) d t+\sigma_{y}^{\mathrm{HJM}}(X(t)) d W(t), \\
d X(t) & =\sqrt{A_{y}(X(t))} d W(t)+\left(\theta(t) e_{1}+B_{y}(X(t))\right) d t .
\end{aligned}
$$

Outside of the domain of $\mathcal{A}$ the forward rate process can be characterised as a mild solution of Equation (3.8) For the concepts of mild, weak, and strong solutions of SPDEs we refer to [13, Section 6.1].

In the one-factor case, the factor process $(X(t))_{t \geq 0}$ is a simple functional of the forward rate. Then Equation (3.8) can be rewritten as an evolution equation for the forward rate process alone (c.f. Equation (5.4)]. This is also possible in the multi-factor case, but the corresponding functional is more complicated, which is why we choose to present the HJM equation in the form 3.8 .

Proof. Differentiating formula (3.6) for forward rates with respect to $t$ and $\tau$ and using $\Psi_{y}^{\prime}(0)=-\lambda$, one obtains for each $\tau \geq 0$

$$
\begin{aligned}
d h(t, \tau)=( & -\int_{t}^{t+\tau} \theta(s)\left\langle\Psi_{y}^{\prime \prime}(t+\tau-s), e_{1}\right\rangle d s+\theta(t+\tau)\left\langle\lambda, e_{1}\right\rangle \\
& \left.+\theta(t)\left\langle\Psi_{y}^{\prime}(\tau), e_{1}\right\rangle\right) d t-\left\langle\Psi_{y}^{\prime}(\tau), d X(t)\right\rangle, \\
\mathcal{A} h(t, \tau)=- & \int_{t}^{t+\tau} \theta(s)\left\langle\Psi_{y}^{\prime \prime}(t+\tau-s), e_{1}\right\rangle d s+\theta(t+\tau)\left\langle\lambda, e_{1}\right\rangle \\
& -\Phi_{y}^{\prime \prime}(\tau)-\left\langle\Psi_{y}^{\prime \prime}(\tau), X(t)\right\rangle .
\end{aligned}
$$

Subtracting the equations and cancelling out the integral as well as the term next to it yields

$$
d h(t, \tau)=\left(\mathcal{A} h(t, \tau)+\Phi_{y}^{\prime \prime}(\tau)+\left\langle\Psi_{y}^{\prime \prime}(\tau), X(t)\right\rangle+\theta(t)\left\langle\Psi_{y}^{\prime}(\tau), e_{1}\right\rangle\right) d t-\left\langle\Psi_{y}^{\prime}(\tau), d X(t)\right\rangle .
$$

When $d X(t)$ is replaced by the right-hand side of SDE (3.1), the $\theta(t)$-term cancels out and one obtains for each $\tau \geq 0$

$$
\begin{aligned}
d h(t, \tau)=( & \left.\mathcal{A} h(t, \tau)+\Phi_{y}^{\prime \prime}(\tau)+\left\langle\Psi_{y}^{\prime \prime}(\tau), X(t)\right\rangle-\left\langle\Psi_{y}^{\prime}(\tau), B_{y}(X(t))\right\rangle\right) d t \\
& -\left\langle\Psi_{y}^{\prime}(\tau), \sqrt{A_{y}(X(t))} d W(t)\right\rangle .
\end{aligned}
$$

The symmetric matrix $\sqrt{A_{y}(X(t))}$ can be moved to the other side of the scalar product, and one immediately recognises the volatility $\sigma_{y}^{\mathrm{HJM}}(X(t))$. A direct calculation shows that the drift is equal to $\mu_{y}^{\mathrm{HJM}}(X(t))$. Indeed,

$$
\begin{aligned}
\Phi_{y}^{\prime \prime}+\left\langle\Psi_{y}^{\prime \prime}, x\right\rangle-\left\langle\Psi_{y}^{\prime}, B_{y}(x)\right\rangle & =F_{y}^{\prime} \circ \Psi_{y} \cdot \Psi_{y}^{\prime}+\left\langle R_{y}^{\prime} \circ \Psi_{y} \cdot \Psi_{y}^{\prime}, x\right\rangle-\left\langle\Psi_{y}^{\prime}, B_{y}(x)\right\rangle \\
& =\left\langle\Psi_{y}, A_{y}(x) \Psi_{y}^{\prime}\right\rangle=\mu_{y}^{\mathrm{HJM}}(x),
\end{aligned}
$$

which follows from the relations

$$
F_{y}^{\prime}(u) \cdot v=\left\langle u, a_{y} v\right\rangle+\left\langle v, b_{y}\right\rangle, \quad\left(R_{y}^{i}\right)^{\prime}(u) \cdot v=\left\langle u, \alpha_{y}^{i} v\right\rangle+\left\langle v, \beta_{y}^{i}\right\rangle .
$$


3.7. Forward rates and Hull-White extensions. Relation (3.6) between the forward rate $h(t)$ and the Hull-White extension $\theta$ plays a key role in calibration (and recalibration) of the model. It can be expressed concisely as

$$
h(t)=\mathcal{H}_{y}(\mathcal{S}(t) \theta, X(t)), \quad \mathcal{S}(t) \theta=\mathcal{C}_{y}(h(t), X(t)), \quad \text { for all } t \geq 0,
$$

where $\mathcal{S}(t)$ is the right shift operator, see Assumption 3.4 iii), $\mathcal{H}_{y}$ calculates the initial forward rate curve from the Hull-White extension given parameter $y$, and $\mathcal{C}_{y}$ performs the inverse operation of calibrating a Hull-White extension to an initial forward rate curve. Formally, for each $(t, x, \theta) \in \mathbb{R}_{+} \times \mathbb{X} \times C\left(\mathbb{R}_{+}\right)$, these operators are given by

$$
\begin{aligned}
\mathcal{S}(t) \theta & =\theta(t+\cdot) \in C\left(\mathbb{R}_{+}\right), \\
\mathcal{H}_{y}(\theta, x) & =\ell-\mathcal{I}_{y}(\theta)-\Phi_{y}^{\prime}-\left\langle\Psi_{y}^{\prime}, x\right\rangle \in C^{1}\left(\mathbb{R}_{+}\right), \\
\mathcal{I}_{y}(\theta) & =\int_{0} \theta(s)\left\langle\Psi_{y}^{\prime}(\cdot-s), e_{1}\right\rangle d s \in C^{1}\left(\mathbb{R}_{+}\right) .
\end{aligned}
$$

Note that $\mathcal{H}_{y}$ involves the Volterra integral operator $\mathcal{I}_{y}$. The operator $\mathcal{C}_{y}$ (the letter $\mathcal{C}$ standing for calibration) is defined as the partial inverse of $\mathcal{H}_{y}$ given by the following theorem.

Theorem 3.6 (Calibration to initial forward rate curves). Let $(h, x) \in C^{1}\left(\mathbb{R}_{+}\right) \times \mathbb{X}$ satisfy $h(0)=\ell+\langle\lambda, x\rangle$. Then the Volterra integral equation $h=\mathcal{H}_{y}(\theta, x)$ has a unique solution $\theta \in C\left(\mathbb{R}_{+}\right)$, which we denote by $\mathcal{C}_{y}(h, x)$.

The theorem is a direct consequence of the following lemma.

Lemma 3.7. For each $y \in \mathbb{Y}$, the Volterra integral operator

$$
\mathcal{I}_{y}: C\left(\mathbb{R}_{+}\right) \rightarrow\left\{h \in C^{1}\left(\mathbb{R}_{+}\right): h(0)=0\right\}
$$

is bijective.

Proof. This follows from [8, Theorem 2.1.8], noting that the integral kernel

$$
K_{y}(s, t)=\left\langle\Psi_{y}^{\prime}(t-s), e_{1}\right\rangle, \quad \text { for all } t \geq s \geq 0,
$$

satisfies $\left|K_{y}(t, t)\right|=\left|\left\langle\lambda, e_{1}\right\rangle\right|>0$ and both $K_{y}$ and $\partial_{t} K_{y}$ are continuous.

Note that calibration of a Hull-White extension $\theta$ requires the inversion of the Volterra integral operator $\mathcal{I}_{y}$. Here the assumption $\left\langle\lambda, e_{1}\right\rangle \neq 0$ is needed.

3.8. Numerical solution of the Volterra equation. In the absence of analytical formulas, the Volterra equation has to be solved numerically. We are aiming at a second order approximation to keep the global error of the simulation scheme of order one. Thus, we approximate the Volterra integral operator $\mathcal{I}_{y}$ by the trapezoid rule, which yields an operator $\widehat{\mathcal{I}}_{y}$ given by

$$
\widehat{\mathcal{I}}_{y}(\theta)\left(\tau_{n}\right)=\delta\left(\frac{1}{2}\left\langle\Psi_{y}^{\prime}\left(\tau_{n}\right), e_{1}\right\rangle \theta(0)+\sum_{i=1}^{n-1}\left\langle\Psi_{y}^{\prime}\left(\tau_{n}-\tau_{i}\right), e_{1}\right\rangle \theta\left(\tau_{i}\right)+\frac{1}{2}\left\langle\Psi_{y}^{\prime}(0), e_{1}\right\rangle \theta\left(\tau_{n}\right)\right),
$$

for each $n \in \mathbb{N}^{+}$, where $\tau_{n}=n \delta$ constitutes a uniform grid of step size $\delta>0$. Approximate solutions $\widehat{\theta}$ can be constructed by solving for continuous piecewise linear (i.e. linear on each interval $\left.\left[\tau_{n}, \tau_{n+1}\right]\right)$ functions $\widehat{\theta}$ satisfying

$$
\widehat{\theta}(0)=\frac{g^{\prime}(0)}{\left\langle\Psi_{y}^{\prime}(0), e_{1}\right\rangle}, \quad \widehat{\mathcal{I}}_{y}(\widehat{\theta})\left(\tau_{n}\right)=g\left(\tau_{n}\right), \quad \text { for all } n \in \mathbb{N}^{+}
$$


As $\widehat{\mathcal{I}}_{y}$ is a second order approximation of $\mathcal{I}_{y}$, it is not surprising that $\widehat{\theta}$ is a second order approximation of $\theta$.

Lemma 3.8. Let $(x, y) \in \mathbb{X} \times \mathbb{Y}$ and $g: \mathbb{R}_{+} \rightarrow \mathbb{R}$ piecewise $C^{4}$ with continuous second derivatives. If $g(0)=0$, then there is a unique piecewise linear function $\widehat{\theta} \in C\left(\mathbb{R}_{+}\right)$ satisfying (3.10). Moreover, $\widehat{\theta}$ is a second order approximation of the exact solution $\theta$ of the Volterra equation $\mathcal{I}_{y}(\theta)=g$ in the sense that for each $T \in \mathbb{R}_{+}$,

$$
\sup _{t \in[0, T]}|\widehat{\theta}(t)-\theta(t)| \leq C \delta^{2},
$$

where $C$ is a constant depending only on $T$ and $g$.

The lemma will be used to show the numerical invertibility of the calibration operator $\mathcal{H}_{y}(\cdot, x)$. In this context, the smoothness assumption on the right-hand side $g$ of the Volterra equation is satisfied if the yields are interpolated sufficiently smoothly.

Proof. This follows from 25. Theorem 3] and 8. Example 2.4.5 and Theorems 2.4.5, 2.4.8], noting that the integral kernel $(3.9)$ is $C^{4}$ and strictly bounded away from zero along the diagonal by our assumption $\left\langle\Psi_{y}^{\prime}(0), e_{1}\right\rangle=\left\langle\lambda, e_{1}\right\rangle \neq 0$.

Note that solving for $\widehat{\theta}$ can be performed efficiently because 3.10 is a linear system for $\left(\widehat{\theta}\left(\tau_{0}\right), \ldots, \widehat{\theta}\left(\tau_{n}\right)\right)$ of lower triangular form.

3.9. Estimation of the affine coefficients. We first discuss how, in principle, the affine coefficients can be identified from covariations of yields and then present some practical considerations on the construction of estimators.

Let $r(t, \tau)$ denote the yield of a zero-coupon bond held from $t$ to $t+\tau$, i.e.

$$
r(t, \tau)=-\frac{1}{\tau} \log P(t, t+\tau), \quad \text { for all } t, \tau \in \mathbb{R}_{+} .
$$

Then by Equation (3.5) the quadratic covariation of yields with maturity $\tau_{i}$ and $\tau_{j}$ satisfies

$$
\frac{d}{d t}\left[r\left(\cdot, \tau_{i}\right), r\left(\cdot, \tau_{j}\right)\right](t)=\frac{1}{\tau_{i} \tau_{j}} \Psi_{y}\left(\tau_{i}\right)^{\top}\left(a_{y}+\sum_{k=1}^{d} \alpha_{y}^{k} X^{k}(t)\right) \Psi_{y}\left(\tau_{j}\right) .
$$

Assume that the left-hand side of $(3.12)$ is known as a function of $\tau_{i}$ and $\tau_{j}$, and that the components of $\Psi_{y}$ are functionally independent. Then the components of $\Psi_{y}$ and the matrix $A_{y}(X(t))$ can be identified. The function $\Psi_{y}$ usually determines the coefficients $\alpha_{y}$ and $\beta_{y}$ uniquely (see Equation (3.4b) . Furthermore, taking account of the admissibility conditions on the matrices $a_{y}$ and $\alpha_{y}^{k}$ (see 15 . Definition 2.6]) one can identify the $\mathbb{R}_{+}$-valued components of $X(t)$ and the matrix $a_{y}$.

Note that (3.12) is derived solely from the diffusion coefficient of the yield dynamics and therefore is invariant under Girsanov's change of measure. Thus, the coefficients $a_{y}, \alpha_{y}, \beta_{y}$ can be estimated from real world observations without specifying the market price of risk. Of course the market price of risk enters as a bias in the estimation, but the estimators do not depend on it. Moreover, under the model hypothesis the estimates do not depend on the choice of $\tau_{i}, \tau_{j}$, which provides a means to reject ill-suited models.

The remaining $\mathbb{R}$-valued components of $X(t)$ and the coefficient $b_{y}$ do not appear in the quadratic covariations $(3.12)$. We now discuss how they can be estimated. First, note that for one-factor models $b_{y}$ is redundant and can be normalised to zero thanks to the Hull-White extension. In the multi-factor case only the first component of the 
vector $b_{y}$ is redundant. Second, note that the short end of the forward rate curve gives a scalar condition on $X(t)$, which allows one to fully identify $X(t)$ if $X(t)$ has only a single $\mathbb{R}$-valued component. In the general multi-factor case, however, some components of $b_{y}$ and $X(t)$ remain undetermined. They may be calibrated to the prevailing market yield curve by regression methods. Alternatively, they may be estimated by econometric methods. However, these require a market price of risk specification. We do not discuss this topic here and refer to 22 for further details.

In practise, the quadratic covariation (3.12) must be estimated from yields $\widehat{r}\left(t_{n}, \tau_{i}\right)$ given by the market for times $t_{n}=n \delta$ and times to maturity $\tau_{i}$. A naive estimator is the realised covariation, which is defined as

$$
\left[\widehat{r}\left(\cdot, \tau_{i}\right), \widehat{r}\left(\cdot, \tau_{j}\right)\right]\left(t_{n}\right)=\sum_{k=1}^{n}\left(\widehat{r}\left(t_{k}, \tau_{i}\right)-\widehat{r}\left(t_{k-1}, \tau_{i}\right)\right)\left(\widehat{r}\left(t_{k}, \tau_{j}\right)-\widehat{r}\left(t_{k-1}, \tau_{j}\right)\right) .
$$

Here also other estimators such as, e.g., Fourier estimators, as introduced by Paul Malliavin and Maria-Elvira Mancino, could be used, see, e.g., 12 for some recent developments. Fixing a time window of length $M$ and a time $t_{n}$, one has

$$
\begin{array}{r}
\frac{\left[\widehat{r}\left(\cdot, \tau_{i}\right), \widehat{r}\left(\cdot, \tau_{j}\right)\right]\left(t_{n}\right)-\left[\widehat{r}\left(\cdot, \tau_{i}\right), \widehat{r}\left(\cdot, \tau_{j}\right)\right]\left(t_{n-M}\right)}{t_{n}-t_{n-M}} \approx \frac{1}{\tau_{i} \tau_{j}} \Psi_{y}\left(\tau_{i}\right)^{\top} a_{y} \Psi_{y}\left(\tau_{j}\right) \\
+\frac{\delta}{\tau_{i} \tau_{j}\left(t_{n}-t_{n-M}\right)} \sum_{k=1}^{d} \Psi_{y}\left(\tau_{i}\right)^{\top} \alpha_{y}^{k} \Psi_{y}\left(\tau_{j}\right) \sum_{m=n-M+1}^{n} X^{k}\left(t_{m}\right) .
\end{array}
$$

Therefore, for any time $t_{n}$ and any selection of times to maturity $\tau_{i}, \tau_{j}$, estimators $\widehat{a}_{y}, \widehat{\alpha}_{y}$, $\widehat{\beta}_{y}, \widehat{X}^{1}\left(t_{n}\right), \ldots, \widehat{X}^{d_{1}}\left(t_{n}\right)$ can be constructed by solving for the best fit in Equation (3.13)

\section{Consistent Recalibration of AfFine Short Rate models}

4.1. Overview. The constant parameter process $y$ of the previous section is now replaced by a stochastic process $Y=(Y(t))_{t \geq 0}$. The situation is particularly simple when $Y$ is piecewise constant. In this case, the Hull-White extension is recalibrated to the prevailing yield curve (i.e., the yield curve given by the model with old parameters) each time the parameter process changes. Later on, the concepts are generalised to arbitrary parameter processes $Y$, resulting in our definition of general CRC models. Geometrically, these models "locally look like" Hull-White extended affine short rate models with fixed parameter $y$. This is made precise in Section 4.8. A semigroup point of view is taken in Section 4.10, leading to an interpretation of CRC models with piecewise constant $Y$ as splitting schemes with respect to a time grid for more general CRC models.

4.2. Setup and notation. We recall the notion of admissible parameters from Assumption 3.1. For all $(s, x) \in \mathbb{R}_{+} \times \mathbb{X}$ and all admissible parameters $(y, \theta) \in \mathbb{Y} \times C\left(\mathbb{R}_{+}\right)$, we let $X=X_{y, \theta}^{s, x}$ denote the unique solution on $[s, \infty)$ of the SDE (3.1) with $\theta(t)$ replaced by $\theta(t-s)$ and initial condition $X(s)=x$. We assume that $\mathbb{H}$ is a Hilbert space of forward rate curves satisfying Assumption 3.4 simultaneously for all $y \in \mathbb{Y}$. We fix a strictly increasing sequence of non-negative deterministic times $\left(t_{n}\right)_{n \in \mathbb{N}_{0}}$.

4.3. CRC models with piecewise constant parameter process. We assume that the parameter process $Y$ is piecewise constant, i.e. for each $t \in\left[t_{n}, t_{n+1}\right)$ we have $Y(t)=Y\left(t_{n}\right)$. 
Definition 4.1 (CRC models with piecewise constant $Y)$. A stochastic process $(h, X, Y)$ with values in $\mathbb{H} \times \mathbb{X} \times \mathbb{Y}$ is called a $C R C$ model if there exists a stochastic process $\theta$ with values in $C\left(\mathbb{R}_{+}\right)$such that the following conditions are satisfied, for each $n \in \mathbb{N}_{0}$ :

(i) The Hull-White extension $\theta$ on $\left[t_{n}, t_{n+1}\right]$ is determined by calibration to $h\left(t_{n}\right)$ :

$$
h\left(t_{n}\right)(0)=\ell+\left\langle\lambda, X\left(t_{n}\right)\right\rangle, \quad \theta\left(t_{n}\right)=\mathcal{C}_{Y\left(t_{n}\right)}\left(h\left(t_{n}\right), X\left(t_{n}\right)\right),
$$

and for $t \in\left[t_{n}, t_{n+1}\right]$

$$
\theta(t)=\mathcal{S}\left(t-t_{n}\right) \theta\left(t_{n}\right)
$$

(ii) The evolution of $X$ on $\left[t_{n}, t_{n+1}\right]$ corresponds to the Hull-White extended affine model determined by the parameters $\left(Y\left(t_{n}\right), \theta\left(t_{n}\right)\right)$ :

$$
X(t)=X_{Y\left(t_{n}\right), \theta\left(t_{n}\right)}^{t_{n}, X\left(t_{n}\right)}(t), \quad t \in\left[t_{n}, t_{n+1}\right],
$$

where $X_{y, \theta}^{s, x}$ is the solution operator of SDE 3.1 defined in Section 4.2 Here, Assumption 3.1 is assumed to hold for the parameters $\left(Y\left(t_{n}\right), \theta\left(t_{n}\right)\right) \in \mathbb{Y} \times C\left(\mathbb{R}_{+}\right)$.

(iii) The evolution of $h$ on $\left[t_{n}, t_{n+1}\right]$ is determined by $X$ according to the prevailing Hull-White extended affine model:

$$
h(t)=\mathcal{H}_{Y\left(t_{n}\right)}(\theta(t), X(t)), \quad t \in\left[t_{n}, t_{n+1}\right] .
$$

We use the same symbols $h$ and $X$ as in Equation (3.8) to denote CRC models. The abuse of notation is motivated by the fact that $(h, X)$ evolves on $\left[t_{n}, t_{n+1}\right]$ according to (3.8) with parameters $\left(Y\left(t_{n}\right), \theta\left(t_{n}\right)\right)$. Note that the process $X$ in Definition 4.1 is continuous because closed intervals $\left[t_{n}, t_{n+1}\right]$ are used in point (ii). We emphasise that the recalibration step (i) happens on a discrete time scale because the parameter process $Y$ is constant on each $\left[t_{n}, t_{n+1}\right]$. By construction the process $(h, X)$ is continuous at each time $t_{n}$.

4.4. Simulation. If we assume that a stochastic model for the evolution of the parameter process $Y$ is specified, one can sample $Y$ on the time grid $\left(t_{n}\right)_{n \in \mathbb{N}_{0}}$. Then CRC models as in Definition 4.1 can be simulated by applying iteratively steps (i)-(iii).

Algorithm 4.2 (Simulation). Given $h\left(t_{0}\right)$ and the process $Y$, calculate $(h, X, Y, \theta)$ on the time grid $\left(t_{n}\right)_{n \in \mathbb{N}_{0}}$ by iteratively executing steps (ii)-(iii) of Definition 4.1. Abort with an error if the assumption in step (iii) is not satisfied, for any $n \in \mathbb{N}_{0}$.

The algorithm is illustrated in Figure 4.1. Note that the forward rate increments are calculated from increments of the affine factor process $X$, which can typically be simulated with high orders of accuracy and proper treatment of boundary conditions. These advantages are thanks to the affine structure of the CRC increments and are not available for general HJM models.

4.5. Efficient updating of forward rate curves. Updating the curve of forward rates as prescribed by Definition 4.1 (iii) involves calculating integrals on time intervals $[0, \tau]$, for large values of $\tau$ (see Section 3.7 for the formulas). A significant speed-up can be obtained when this update is done using the alternative formula provided by the following lemma, which involves only integrals over time intervals of length $\delta=t_{n+1}-t_{n}$. 


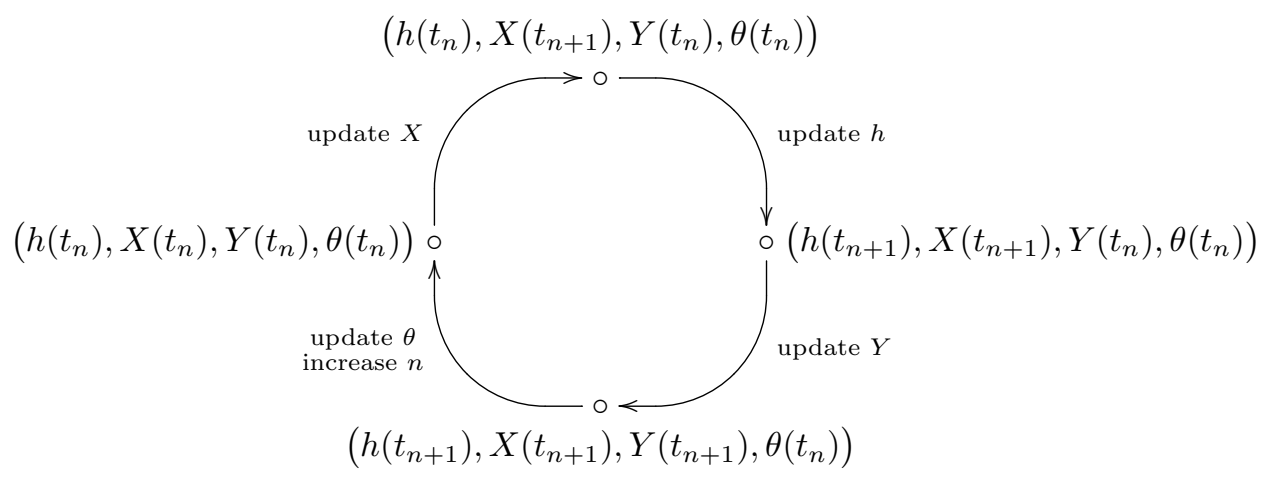

Figure 4.1. Simulation of CRC models. Updating $\theta, X, h$ is done using (ii), (ii), (iii) of Definition 4.1, respectively. Updating $Y$ is done using the exogenously given model for $Y$.

Lemma 4.3 (Efficient updating of forward rate curves). Definition 4.1 iiii) can be rewritten as

$$
\begin{aligned}
h\left(t_{n+1}\right)=\mathcal{S}(\delta) h\left(t_{n}\right) & +\mathcal{S}(\delta) \Phi_{Y\left(t_{n}\right)}^{\prime}-\Phi_{Y\left(t_{n}\right)}^{\prime}+\left\langle\mathcal{S}(\delta) \Psi_{Y\left(t_{n}\right)}^{\prime}, X\left(t_{n}\right)\right\rangle \\
& -\left\langle\Psi_{Y\left(t_{n}\right)}^{\prime}, X\left(t_{n+1}\right)\right\rangle+\int_{0}^{\delta} \theta\left(t_{n}\right)(s)\left\langle\mathcal{S}(\delta-s) \Psi_{Y\left(t_{n}\right)}^{\prime}, e_{1}\right\rangle d s
\end{aligned}
$$

where $\delta=t_{n+1}-t_{n}$.

Proof. By conditions (ii) and (iii) of Definition 4.1.

$$
\begin{aligned}
h\left(t_{n+1}\right)-\mathcal{S}(\delta) h\left(t_{n}\right)= & \mathcal{H}_{Y\left(t_{n}\right)}\left(\mathcal{S}(\delta) \theta\left(t_{n}\right), X\left(t_{n+1}\right)\right)-\mathcal{S}(\delta) \mathcal{H}_{Y\left(t_{n}\right)}\left(\theta\left(t_{n}\right), X\left(t_{n}\right)\right) \\
= & \ell-\mathcal{I}_{Y\left(t_{n}\right)}\left(\mathcal{S}(\delta) \theta\left(t_{n}\right)\right)-\Phi_{Y\left(t_{n}\right)}^{\prime}-\left\langle\Psi_{Y\left(t_{n}\right)}^{\prime}, X\left(t_{n+1}\right)\right\rangle \\
& -\ell+\mathcal{S}(\delta) \mathcal{I}_{Y\left(t_{n}\right)}\left(\theta\left(t_{n}\right)\right)+\mathcal{S}(\delta) \Phi_{Y\left(t_{n}\right)}^{\prime}\left\langle\mathcal{S}(\delta) \Psi_{Y\left(t_{n}\right)}^{\prime}, X\left(t_{n}\right)\right\rangle .
\end{aligned}
$$

Now the assertion of the lemma follows from the relationships

$$
\mathcal{S}(\delta) \mathcal{I}_{y}(\theta)-\mathcal{I}_{y}(\mathcal{S}(\delta) \theta)=\int_{0}^{\delta} \theta(s)\left\langle\mathcal{S}(\delta-s) \Psi_{y}^{\prime}, e_{1}\right\rangle d s, \quad \text { for all }(\delta, \theta) \in \mathbb{R}_{+} \times C\left(\mathbb{R}_{+}\right),
$$

which can easily be verified from the definition.

\subsection{Bond prices and forward rates.}

Theorem 4.4 (Zero-coupon bond price and forward rate). Let $(h, X, Y)$ be a CRC model as in Definition 4.1 with corresponding process $\theta$. Define

$$
P(t, T)=e^{-\int_{t}^{T} h(t, s-t) d s}, \quad r(t)=h(t, 0)=\ell+\langle\lambda, X(t)\rangle, \quad B(t)=e^{\int_{0}^{t} r(s) d s} .
$$

Then the discounted price process $t \mapsto P(t, T) / B(t)$ is a $\mathbb{P}$-local martingale, for each $T \geq 0$. In this sense, the bond market is free of arbitrage. Moreover, the following affine bond 
pricing formulas hold:

$$
\begin{aligned}
\log (P(t, T))= & -\ell(T-t)+\int_{0}^{T-t} \theta(t)(s)\left\langle\Psi_{Y(t)}(T-t-s), e_{1}\right\rangle d s \\
& +\Phi_{Y(t)}(T-t)+\left\langle\Psi_{Y(t)}(T-t), X(t)\right\rangle, \\
h(t, \tau)= & \ell-\int_{0}^{\tau} \theta(t)(s)\left\langle\Psi_{Y(t)}^{\prime}(\tau-s), e_{1}\right\rangle d s-\Phi_{Y(t)}^{\prime}(\tau)-\left\langle\Psi_{Y(t)}^{\prime}(\tau), X(t)\right\rangle .
\end{aligned}
$$

Note: the following proof shows the stronger statement that discounted bond prices are true martingales.

Proof. On each interval $\left[t_{n}, t_{n+1}\right]$, the evolution of forward rate curves $h(t)$ stems from a Hull-White extended affine short rate model. Therefore, for each $T \geq 0$, the discounted price process $t \mapsto P(t, T) / B(t)$ is a martingale on each interval $\left[t_{n}, t_{n+1}\right]$. Moreover, the process is continuously concatenated at the boundaries $t_{n}$ of the intervals. It follows that the process is a martingale on $[0, \infty)$. The affine bond pricing formulas are equivalent to $h(t)=\mathcal{H}_{Y(t)}(\theta(t), X(t))$, which holds by Definition 4.1.iii).

\subsection{Heath-Jarrow-Morton equation.}

Theorem 4.5 (HJM equation). Let $(h, X, Y)$ be a CRC model as in Definition 4.1 with corresponding process $\theta$ and assume that $h(t) \in \mathcal{D}(\mathcal{A})$, for each $t \geq 0$. Then the following properties hold:

(i) the expression $\mathcal{C}_{Y(t)}(h(t), X(t))$ is well-defined and equals $\theta(t)$, for all $t \geq 0$;

(ii) the parameters $(Y(t), \theta(t))$ are admissible, for all $t \geq 0$; and

(iii) the process $(h, X)$ is a strong solution of the following SPDE on $\mathbb{H} \times \mathbb{X}$ :

$$
\begin{aligned}
d h(t) & =\left(\mathcal{A} h(t)+\mu_{Y(t)}^{\mathrm{HJM}}(X(t))\right) d t+\sigma_{Y(t)}^{\mathrm{HJM}}(X(t)) d W(t), \\
d X(t) & =\sqrt{A_{Y(t)}(X(t))} d W(t)+\left(\mathcal{C}_{Y(t)}(h(t), X(t))(0) e_{1}+B_{Y(t)}(X(t))\right) d t .
\end{aligned}
$$

Proof. This follows from Definition 4.1 and Theorem 3.5.

4.8. Geometric interpretation. The consistent recalibration scheme has a nice geometric interpretation. Forward rate curves of a Hull-White extended affine short rate model remain within the finite dimensional manifold with boundary given by

$$
\left\{-\int_{0}^{\tau} \theta(t+s)\left\langle\Psi_{y}^{\prime}(\tau-s), e_{1}\right\rangle d s+\ell-\Phi_{y}^{\prime}(\tau)-\left\langle\Psi_{y}^{\prime}(\tau), x\right\rangle \mid(t, x) \in \mathbb{R}_{+} \times \mathbb{R}^{d}\right\},
$$

as can be seen from Theorem 3.3. These submanifolds foliate the space of forward rate curves or large portions thereof. Let $h$ be a forward rate curve. Then, for every choice of functional characteristics $\left(F_{y}, R_{y}\right)$, there is at most one leaf through $h$. However, if $\left(F_{y}, R_{y}\right)$ is allowed to vary, there are in general many leaves through $h$. A choice of leaf corresponds to a choice of foliation and thus to a choice functional characteristics $\left(F_{y}, R_{y}\right)$.

A CRC model is constructed by concatenating forward rate evolutions on leaves belonging to different foliations. This allows the otherwise constant coefficients $\left(F_{y}, R_{y}\right)$ to change over time. The result is an HJM model which is "tangent" to Hull-White extended affine short rate models. This is illustrated in Figure 4.2. 

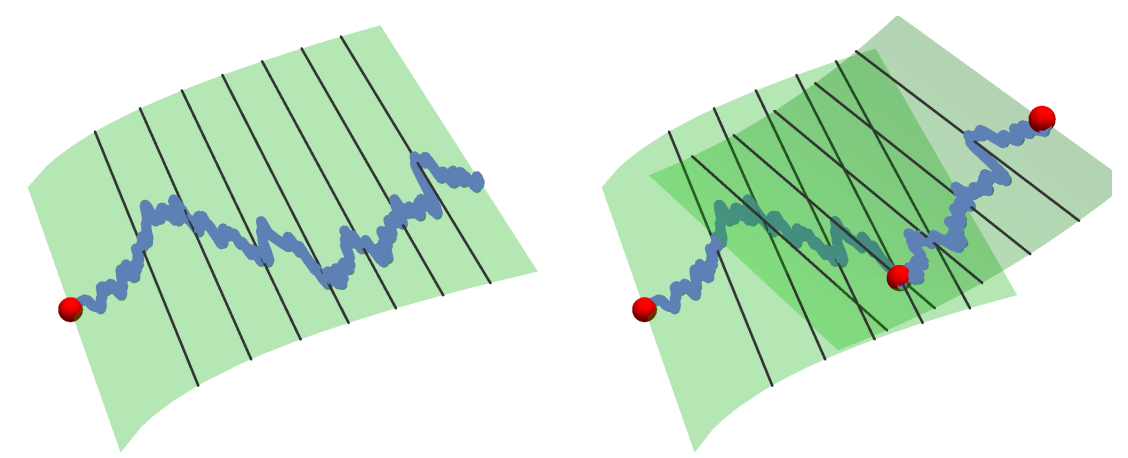

FiguRE 4.2. Each affine short rate model foliates the space of forward rate curves into invariant leaves. CRC models are concatenations of forward rate evolutions belonging to different foliations or limits of such concatenations.

4.9. CRC models. We generalise CRC models of Section 4.3 to arbitrary parameter processes $Y$. In this section $Y$ is not required to be piecewise constant as in the last sections. To characterise such models, we use the SPDE derived in Theorem 4.5.

Definition 4.6 (CRC models). A $C R C$ model is a process $(h, X, Y)$ with values in $\mathbb{H} \times \mathbb{X} \times \mathbb{Y}$ satisfying conditions (ii)-(iii) of Theorem 4.5 with $\theta(t)=\mathcal{C}_{Y(t)}(h(t), X(t))$ for each $t \geq 0$.

We may think of CRC models as continuous-time limits of the concatenations described in Section 4.8. Note that these models satisfy all conclusions of Theorem 4.4 they are free of arbitrage because discounted bond prices $P(t, T) / B(t)$ are local martingales thanks to the HJM drift condition [17, Theorem 6.1], and the short rate can be written as $r(t)=h(t)(0)=\ell+\langle\lambda, X(t)\rangle$.

4.10. Semigroup interpretation. Assume that the parameter process $Y$ is Markov on $\mathbb{Y}$, and that the SPDE (4.2) has a unique mild solution which depends continuously on the initial condition. Then the CRC model $(h, X, Y)$ in Definition 4.6 is Markov on $\mathbb{H} \times \mathbb{X} \times \mathbb{Y}$ 13. c.f. Theorem 9.14]. Let $\mathcal{P}=(\mathcal{P}(t))_{t>0}$ denote the corresponding semigroup on the Banach space $C_{b}(\mathbb{H} \times \mathbb{X} \times \mathbb{Y})$ of bounded continuous functions, i.e.,

$$
\mathcal{P}(t) f\left(h_{0}, x_{0}, y_{0}\right)=\mathbb{E}\left[f(h(t), X(t), Y(t)) \mid(h(0), X(0), Y(0))=\left(h_{0}, x_{0}, y_{0}\right)\right]
$$

Moreover, let $\mathcal{Q}=(\mathcal{Q}(t))_{t \geq 0}$ denote the semigroup on $C_{b}(\mathbb{H} \times \mathbb{X} \times \mathbb{Y})$ obtained by holding the parameter process $Y(\bar{t}) \equiv y$ fixed, i.e.,

$$
\mathcal{Q}(t) f\left(h_{0}, x_{0}, y_{0}\right)=\mathbb{E}\left[f\left(h(t), X(t), y_{0}\right) \mid(h(0), X(0))=\left(h_{0}, x_{0}\right)\right],
$$

where $(h, X)$ are as in Theorem 3.5 with $y=y_{0}$. Finally, let $\mathcal{R}=(\mathcal{R}(t))_{t \geq 0}$ denote the semigroup on $C_{b}(\mathbb{H} \times \mathbb{X} \times \mathbb{Y})$ describing the evolution of $Y$, i.e.,

$$
\mathcal{R}(t) f\left(h_{0}, x_{0}, y_{0}\right)=\mathbb{E}\left[f\left(h_{0}, x_{0}, Y(t)\right) \mid Y(0)=y_{0}\right]
$$

Then, the concatenation $(\mathcal{R}(\delta) \mathcal{Q}(\delta))^{n} f$ of semigroups describes CRC models with a piecewise constant parameter process as in Definition 4.1. Indeed,

$$
(\mathcal{R}(\delta) \mathcal{Q}(\delta))^{n} f\left(h_{0}, x_{0}, y_{0}\right)=\mathbb{E}\left[h\left(t_{n}\right), X\left(t_{n}\right), Y\left(t_{n}\right)\right], \quad \text { for all } n \in \mathbb{N}_{0},
$$


where $\delta=t_{n+1}-t_{n}$ is the step size, $\left(h\left(t_{n}\right), X\left(t_{n}\right)\right)$ is obtained by executing the simulation scheme of Section 4.4 and $Y\left(t_{n}\right)$ by sampling the Markov process $Y$ on that time grid.

4.11. Simulation of CRC models by splitting schemes. The semigroup interpretation of Section 4.10 allows one to view Algorithm 4.2 as an exponential Euler splitting scheme for general CRC models as in Definition 4.6. To see this, let $f: \mathbb{H} \times \mathbb{X} \times \mathbb{Y} \rightarrow \mathbb{R}$ be twice differentiable with derivatives being uniformly continuous on bounded sets and assume that $\mathbb{H} \subseteq \operatorname{dom}(\mathcal{A})$. Then, under appropriate assumptions on $Y$, Itō's formula holds for $f(h(t), X(t), Y(t))$ by [13. Theorem 4.17]. It follows that $f$ lies in the common domain of the generators $\mathcal{G}^{\mathcal{P}}, \mathcal{G}^{\mathcal{Q}}, \mathcal{G}^{\mathcal{R}}$ of the semigroups $\mathcal{P}, \mathcal{Q}, \mathcal{R}$, and, if $Y$ is independent of $W$,

$$
\mathcal{G}^{\mathcal{P}} f=\mathcal{G}^{\mathcal{Q}} f+\mathcal{G}^{\mathcal{R}} f .
$$

The exponential Euler splitting scheme with respect to this splitting is defined as

$$
\mathcal{P}(n \delta) f \approx\left(\exp \left(\delta \mathcal{G}^{\mathcal{R}}\right) \exp \left(\delta \mathcal{G}^{\mathcal{Q}}\right)\right)^{n} f=(\mathcal{R}(\delta) \mathcal{Q}(\delta))^{n} f, \quad \text { for all } n \in \mathbb{N}_{0} .
$$

By the considerations in Section 4.10 it coincides with the simulation scheme of Section 4.4 The advantages of this simulation scheme in comparison to other methods are discussed in Sections 2.2 and 4.13 .

4.12. Calibration of CRC models. In order to calibrate CRC models we need to estimate a time series of the parameter process $Y$ from market data, and fit a model for this time series. Estimating a time series of the parameter process $Y$ can be done as explained in Section 3.9 for Hull-White extended affine models. The resulting time series $Y$ consists of model parameters under a risk neutral measure, but they can be estimated from real world observations since the estimators are obtained solely from the volatility of the forward rate process.

Calibrating CRC models requires the additional task of selecting and fitting a model for the estimated time series of $Y$. This completes the model specification under a risk neutral probability measure. We do not discuss the market price of risk specification in this paper but refer to 22 for more details.

4.13. Robust calibration, consistency, and analytic tractability. It is time to address the question to what extent CRC models satisfy the interest rate modelling principles set forth in the introduction. First, we formalise the notion of consistency and the consistent recalibration property. We do this for CRC models, but it is unproblematic to generalise the definition to other forward rate models. Let $\mathrm{pr}_{\mathbb{H}}$ denote the projection of $\mathbb{H} \times \mathbb{X} \times \mathbb{Y}$ onto $\mathbb{H}$.

Definition 4.7. Let $(h(t), X(t), Y(t))_{t \geq 0}$ be a CRC model and $\mathcal{I} \subseteq \mathbb{H} \times \mathbb{X} \times \mathbb{Y}$. Then the model is called consistent with $\mathcal{I}$ if $(h(t), X(t), Y(t)) \in \mathcal{I}$ holds with probability one for any $t>0$ and initial condition $\left(h_{0}, x_{0}, y_{0}\right) \in \mathcal{I}$. Moreover, the model satisfies the consistent recalibration property with respect to $\mathcal{I}$ if the support of the law of $h(t)$ contains $\operatorname{pr}_{\mathbb{H}}(\mathcal{I})$ for any $t>0$ and initial condition $\left(h_{0}, x_{0}, y_{0}\right) \in \mathcal{I}$.

The consistent recalibration property is equivalent to any open subset of $\mathrm{pr}_{\mathbb{H}}(\mathcal{I})$ being reached at any time $t>0$ with positive probability. Observe that the consistent recalibration property does not hold on any reasonably large set $\mathcal{I}$ for Hull-White extended affine factor models as in Section 3. Indeed, as explained in Section 4.8, for any given initial value the process $h$ remains within a finite dimensional submanifold of $\mathbb{H}$. CRC models, on the other hand, enjoy the following properties: 
- Robust calibration: the robust calibration principle is satisfied perfectly. Indeed, the method described in Section 4.12 allows us to use the entire present and past market data of yields to select a model. Whenever possible, the parameters are estimated from realised covariations of yields, which allows one to bypass the usual inverse problems in calibration.

Moreover, requiring parameters to remain constant throughout the life time of the model is less restrictive in CRC models than in the underlying affine factor models. The reason is that the parameters of the underlying affine model are turned into state variables of the CRC model.

- Consistency: the canonical state space $\mathcal{I}$ of $\mathrm{CRC}$ models is the subset of $\mathbb{H} \times \mathbb{X} \times \mathbb{Y}$ determined by the admissibility condition on the underlying Hull-White extended affine factor model (see Assumption 3.1). Under sensible specifications of the affine factor model, $\mathcal{I} \cap(\mathbb{H} \times\{x\} \times\{y\})$ is large enough to contain all realistic market curves, for each fixed $(x, y) \in \mathbb{X} \times \mathbb{Y}$. If the Hilbert space $\mathbb{H}$ is continuously embedded in $C^{1}\left(\mathbb{R}_{+}\right)$, then $\mathcal{I}$ is also large in the topological sense of having non-empty interior.

In this setup consistency holds by construction because the state process $(h, X, Y)$ of CRC models does not leave the set $\mathcal{I}$. The consistent recalibration property can be verified using standard arguments: the support of $(h(t), X(t), Y(t))$ is the closure of the reachable set of an associated control problem [27, the reachable set is stable under the flows of the driving vector fields and their Lie brackets, and generically speaking, as soon as there is noise in the parameter process $Y$, these vector fields together with their brackets span dense subspaces of $\mathbb{H}$. The exact conditions are worked out for the Vasiček case in Section 5.7.

- Analytic tractability: the simulation scheme for CRC models Algorithm 4.2 transfers the task of sampling state variable increments to a finite-dimensional setting. Namely, instead of simulating forward rate increments from an infinitedimensional space, it is sufficient to simulate increments of the finite-dimensional processes $X$ and $Y$. This allows one to take advantage of the existing high-order schemes for the simulation of affine processes. Note that all operations in $\mathrm{Al}-$ gorithm 4.2 which involve infinite-dimensional objects are deterministic. The complexity for simulation reduces dramatically when high order methods for finite dimensional equations are applied which are often not at hand for infinite dimensional equations. Additionally often exact schemes are available in the affine finite dimensional setting, e.g., for CIR or Wishart type processes besides of course Gaussian processes.

\section{Consistent Recalibration of Vasiček models}

5.1. Overview. We describe CRC models based on the Hull-White extended Vasiček model in full detail. Moreover, we show using semigroup theory that the simulation scheme of Section 4.4 converges to the CRC model of Definition 4.6 in the continuous-time limit.

5.2. Setup and notation. We use the setup of Sections 3.2 and 4.2 , setting $\mathbb{X}=\mathbb{R}$, $\ell=0, \lambda=1$. We do not specify the parameter space $\mathbb{Y}$, yet, but we assume that for each $(x, y) \in \mathbb{X} \times \mathbb{Y}$, the volatility and drift coefficients are given by $A_{y}(x)=a_{y} \in[0, \infty)$ and $B_{y}(x)=\beta_{y} x$ with $\beta_{y} \in(-\infty, 0)$. For simplicity, we choose equidistant grids of times $t_{n}=n \delta$ and times to maturity $\tau_{n}=n \delta$, for all $n \in \mathbb{N}_{0}$, where $\delta$ is a positive constant. 
5.3. Hull-White extended Vasiček models. For each parameter $(y, \theta) \in \mathbb{Y} \times C\left(\mathbb{R}_{+}\right)$, the SDE for the short rate process is

$$
d r(t)=\left(\theta(t)+\beta_{y} r(t)\right) d t+\sqrt{a_{y}} d W(t),
$$

where $W$ is one-dimensional $(\mathcal{F}(t))_{t \geq 0}$-Brownian motion. Assumption 3.1 is satisfied for each parameter $(y, \theta)$. The functional characteristics $(F, R)$ from Section 3.4 are

$$
F_{y}(u)=\frac{a_{y}}{2} u^{2}, \quad R_{y}(u)=\beta_{y} u, \quad \text { for all } u \in \mathbb{R},
$$

and the solutions $\left(\Phi_{y}, \Psi_{y}\right)$ of the corresponding Riccati equations are

$$
\Phi_{y}(t)=\frac{a_{y}}{4 \beta_{y}^{3}}\left(2 \beta_{y} t-4 e^{\beta_{y} t}+3+e^{2 \beta_{y} t}\right), \quad \Psi_{y}(t)=\frac{1}{\beta_{y}}\left(1-e^{\beta_{y} t}\right), \quad \text { for all } t \geq 0 .
$$

By Theorem 3.3, the forward rates in the Hull-White extended Vasiček model (5.1) with fixed parameters $(y, \theta)$ are given by $h(t)=\mathcal{H}_{y}(\mathcal{S}(t) \theta, r(t)) \in C^{1}\left(\mathbb{R}_{+}\right)$, where

$$
\mathcal{H}_{y}(\theta, x)(\tau)=\int_{0}^{\tau} \theta(s) e^{\beta_{y}(\tau-s)} d s-\frac{a_{y}}{2 \beta_{y}^{2}}\left(1-e^{\beta_{y} \tau}\right)^{2}+e^{\beta_{y} \tau} x,
$$

for all $(x, \theta, \tau) \in \mathbb{R} \times C\left(\mathbb{R}_{+}\right) \times \mathbb{R}_{+}$. Due to the simple structure of the integral kernel $e^{\beta_{y}(\tau-s)}$, there is a closed-form expression for the calibration operator,

$$
\mathcal{C}_{y}(h)(\tau)=h^{\prime}(\tau)-\beta_{y} h(\tau)-\frac{a_{y}}{2 \beta_{y}}\left(1-e^{2 \beta_{y} \tau}\right), \quad \text { for all }(h, \tau) \in C^{1}\left(\mathbb{R}_{+}\right) \times \mathbb{R}_{+} .
$$

This can be verified using the definitions. Note that the calibration operator does not depend on $x$. Therefore, we dropped $x$ from the notation $\mathcal{C}_{y}(h, x)$.

The HJM drift and volatility from Section 3.6 are

$$
\mu_{y}^{\mathrm{HJM}}(\tau)=-\frac{a_{y}}{\beta_{y}} e^{\beta_{y} \tau}\left(1-e^{\beta_{y} \tau}\right), \quad \sigma_{y}^{\mathrm{HJM}}(\tau)=\sqrt{a_{y}} e^{\beta_{y} \tau}, \quad \text { for all } \tau \in \mathbb{R}_{+} .
$$

Note that these expressions do not depend on $x$, which is why we again dropped $x$ from the previous notation $\mu_{y}^{\mathrm{HJM}}(x)(\tau), \sigma_{y}^{\mathrm{HJM}}(x)(\tau)$. The HJM equation for forward rates then reads as

$$
d h(t)=\left(\mathcal{A} h(t)+\mu_{y}^{\mathrm{HJM}}\right) d t+\sigma_{y}^{\mathrm{HJM}} d W(t) .
$$

5.4. Vasiček CRC models. Since the factor process is a function of the forward rate process, i.e., $X(t)=r(t)=h(t, 0)$, the corresponding CRC models can be characterised by the process $(h, Y)$ instead of $(h, X, Y)$. Thus, in accordance with Theorem 4.5 and Definition 4.6, a process $(h, Y)$ with values in $\mathbb{H} \times \mathbb{Y}$ may be called a CRC model if $h$ satisfies the SPDE

$$
d h(t)=\left(\mathcal{A} h(t)+\mu_{Y(t)}^{\mathrm{HJM}}\right) d t+\sigma_{Y(t)}^{\mathrm{HJM}} d W(t),
$$

with drift $\mu_{Y(t)}^{\mathrm{HJM}}$ and volatility $\sigma_{Y(t)}^{\mathrm{HJM}}$ defined in 5.3 . Beyond the obvious requirement that these quantities are well-defined, for all $t \in \mathbb{R}_{+}$, no further conditions are needed. In other words, the maximally admissible set $\mathcal{I}$ in the Vasiček case is the entire Hilbert space $\mathbb{H}$. 
5.5. Simulation of Vasiček CRC models. Given the parameter process $Y$ with values in $\mathbb{Y}$, the CRC model is simulated as described in Algorithm 4.2. The following observations make the algorithm particularly effective. First, the state process $X$ is a function of the forward rate and can be eliminated as a state variable. Second, the short rate process can be simulated exactly. Indeed, in the model with constant parameter $y, r(t)$ is normally distributed,

$$
r(t) \sim \mathcal{N}\left(e^{\beta_{y} t} r_{0}+\int_{0}^{t} e^{\beta_{y}(t-s)} \theta(s) d s, \frac{a_{y}}{2 \beta_{y}}\left(e^{2 \beta_{y} t}-1\right)\right) .
$$

Third, inverting the Volterra integral operator can be avoided by using closed-form expression $\sqrt{5.2}$ of the calibration operator.

Discretisation is done on the uniform grid $t_{n}=\tau_{n}=\delta n$ for a choice of finitely many times to maturity $\tau_{n}$. Integrals are approximated to second order by the trapezoid rule, which leads to a global error of order one (see Section 5.6 and Section 7.7). The resulting scheme works as follows.

Algorithm 5.1 (Simulation). Given $(h(0), \mathcal{A} h(0))$ and the parameter process $Y$, execute iteratively the following steps, for each $n \in \mathbb{N}_{0}$ :

(i) The values of $\theta\left(t_{n}\right)=\mathcal{C}_{Y\left(t_{n}\right)}\left(h\left(t_{n}\right)\right)$ at times to maturity 0 and $\delta$ are calculated using 5.2 ,

$$
\begin{aligned}
& \theta\left(t_{n}\right)(0)=\mathcal{A} h\left(t_{n}\right)(0)-\beta_{Y\left(t_{n}\right)} h\left(t_{n}\right)(0) \\
& \theta\left(t_{n}\right)(\delta)=\mathcal{A} h\left(t_{n}\right)(\delta)-\beta_{Y\left(t_{n}\right)} h\left(t_{n}\right)(\delta)-\frac{a_{Y\left(t_{n}\right)}}{2 \beta_{Y\left(t_{n}\right)}}\left(1-e^{2 \beta_{Y\left(t_{n}\right)} \delta}\right)
\end{aligned}
$$

and $\mathcal{I}_{Y\left(t_{n}\right)}\left(\theta\left(t_{n}\right)\right)(\delta)$ is approximated by the trapezoid rule as follows:

$$
\widehat{\mathcal{I}}_{Y\left(t_{n}\right)}\left(\theta\left(t_{n}\right)\right)(\delta)=-\frac{\delta}{2}\left(e^{\beta_{Y\left(t_{n}\right)} \delta} \theta\left(t_{n}\right)(0)+\theta\left(t_{n}\right)(\delta)\right) .
$$

(ii) A sample $r\left(t_{n+1}\right)$ is drawn such that conditionally on $\mathcal{F}\left(t_{n}\right), r\left(t_{n+1}\right)$ has normal distribution

$$
r\left(t_{n+1}\right) \sim \mathcal{N}\left(e^{\beta_{Y\left(t_{n}\right)} \delta} h\left(t_{n}\right)(0)-\widehat{\mathcal{I}}_{Y\left(t_{n}\right)}\left(\theta\left(t_{n}\right)\right)(\delta), \frac{a_{\left.Y\left(t_{n}\right)\right)}}{2 \beta_{Y\left(t_{n}\right)}}\left(e^{2 \beta_{Y\left(t_{n}\right)} \delta}-1\right)\right) .
$$

(iii) $\left(h\left(t_{n+1}\right), \mathcal{A} h\left(t_{n+1}\right)\right)$ is calculated from $\left(h\left(t_{n}\right), \mathcal{A} h\left(t_{n}\right), r\left(t_{n+1}\right)\right)$ using Lemma 4.3 .

$$
\begin{aligned}
h\left(t_{n+1}\right)(\tau)=h\left(t_{n}\right)(\delta+\tau)+\frac{a_{Y\left(t_{n}\right)}}{2 \beta_{Y\left(t_{n}\right)}^{2}}\left(\left(1-e^{\beta_{Y\left(t_{n}\right)}(\delta+\tau)}\right)^{2}-\left(1-e^{\beta_{Y\left(t_{n}\right)} \tau}\right)^{2}\right) \\
+e^{\beta_{Y\left(t_{n}\right)} \tau}\left(-e^{\beta_{Y\left(t_{n}\right)} \delta} r\left(t_{n}\right)+r\left(t_{n+1}\right)+\widehat{\mathcal{I}}_{Y\left(t_{n}\right)}\left(\theta\left(t_{n}\right)\right)(\delta)\right), \\
\mathcal{A} h\left(t_{n+1}\right)(\tau)=\mathcal{A} h\left(t_{i}\right)(\delta+\tau) \\
+\frac{a_{Y\left(t_{n}\right)}}{\beta_{Y\left(t_{n}\right)}}\left(e^{\beta_{Y\left(t_{n}\right)} \tau}+e^{2 \beta_{Y\left(t_{n}\right)}(\tau+\delta)}-e^{2 \beta_{Y\left(t_{n}\right)} \tau}-e^{\beta_{Y\left(t_{n}\right)}(\delta+\tau)}\right) \\
+\beta_{Y\left(t_{n}\right)} e^{\beta_{Y\left(t_{n}\right)} \tau}\left(-e^{\beta_{Y\left(t_{n}\right)} \delta} r\left(t_{n}\right)+r\left(t_{n+1}\right)+\widehat{\mathcal{I}}_{Y\left(t_{n}\right)}\left(\theta\left(t_{n}\right)\right)(\delta)\right) .
\end{aligned}
$$

Here, $h\left(t_{n+1}\right)$ must be calculated at all times to maturity $\tau_{i}$, whereas $\mathcal{A} h\left(t_{n+1}\right)$ is needed only at $\tau_{0}=0$ and $\tau_{1}=\delta$. 
5.6. Convergence of the simulation scheme. In this section, we show that scheme of Section 5.5 converges to the CRC model (5.5) as the size $\delta$ of the time grid tends to zero. We are not aiming for the highest generality. Instead, we show how the results follow from standard semigroup theory.

Assumption 5.2. The parameter process $Y$ takes values in $\mathbb{Y}=\mathbb{R}^{p}$ and satisfies

$$
d Y(t)=(A Y(t)+\mu(Y(t))) d t+\sigma(Y(t)) d \widetilde{W}(t),
$$

where $A: \mathbb{R}^{p} \rightarrow \mathbb{R}^{p}$ is a linear mapping generating a semigroup of contractions on $\mathbb{R}^{p}, \mu \in$ $C_{b}^{\infty}\left(\mathbb{R}^{p} ; \mathbb{R}^{p}\right), \sigma \in C_{b}^{\infty}\left(\mathbb{R}^{p}, \mathbb{R}^{p \times q}\right)$, and $\widetilde{W}$ is $q$-dimensional $\mathcal{F}(t)$-Brownian motion, independent of $W$. We write $C_{b}^{\infty}$ for bounded functions with bounded derivatives of all orders. The above SDE has a unique solution for any initial condition $Y(s)=y$, where $(s, y) \in \mathbb{R}_{+} \times \mathbb{Y}$.

Assumption 5.3. The mappings $y \mapsto \sqrt{a_{y}}$ and $y \mapsto \beta_{y}$ are of class $C_{b}^{\infty}\left(\mathbb{R}^{p}\right)$ and $\sup _{y \in \mathbb{Y}} \beta_{y}<0$ holds.

As Vasiček CRC models can be characterised in terms of $(h, Y)$ instead of $(h, X, Y)$, the semigroups $\mathcal{P}, \mathcal{Q}$, and $\mathcal{R}$ from Section 4.10 are now assumed to be defined on $C_{b}(\mathbb{H} \times \mathbb{Y})$ instead of $C_{b}(\mathbb{H} \times \mathbb{X} \times \mathbb{Y})$. Recall that $\mathcal{P}$ describes the joint evolution of the process $(h, Y)$, $\mathcal{Q}$ the evolution of $h$ with $Y$ fixed, and $\mathcal{R}$ the evolution of $Y$ with $h$ fixed.

Theorem 5.4. There exists a separable Hilbert space $\mathbb{H}$ of continuous functions on $\mathbb{R}_{+}$ and a Banach space $\mathbb{B}$ of continuous functions on $\mathbb{H} \times \mathbb{Y}$ such that $\mathcal{P}, \mathcal{Q}$, and $\mathcal{R}$ are strongly continuous semigroups on $\mathbb{B}$. Moreover, for each $T \in \mathbb{R}_{+}$there exists a constant $C$ such that

$$
\left\|\mathcal{P}(t) f-(\mathcal{R}(t / n) \mathcal{Q}(t / n))^{n} f\right\|_{\mathbb{B}} \leq C n^{-1}\|f\|_{\mathbb{B}^{\prime}}, \quad \text { for all } f \in \mathbb{B}^{\prime}, t \in[0, T], n \in \mathbb{N}^{+},
$$

where $\mathbb{B}^{\prime}$ is a Banach space which is densely and continuously embedded in $\mathbb{B}$.

The space $\mathbb{B}^{\prime}$ is large enough to be relevant in applications: any $C^{4}$ function on $\mathbb{H}_{0} \times \mathbb{Y}$ belongs to $\mathbb{B}^{\prime}$, where $\mathbb{H}_{0} \supset \mathbb{H}$ is defined in the proof below.

Proof. We proceed as in 14] and [16]. Let $\left(\gamma_{i}\right)_{i \in \mathbb{N}_{0}}$ be a strictly increasing sequence of real numbers strictly greater than 3 . For each $i \in \mathbb{N}_{0}$, define a separable Hilbert space $\mathbb{H}_{i}$ by

$$
\mathbb{H}_{i}=\left\{h \in L_{\mathrm{loc}}^{1}: h^{(j)} \in L_{\mathrm{loc}}^{1} \text { and } \int_{(0, \infty)} h^{(j)}(\tau)^{2}(1+\tau)^{\gamma_{i}} d \tau<\infty, \forall j=1, \ldots, i+1\right\} \text {, }
$$

where $L_{\text {loc }}^{1}$ denotes the space of locally integrable functions on $(0, \infty)$. Every function in $\mathbb{H}_{0}$ is continuous, bounded and has a well-defined limit $h(\infty)=\lim _{\tau \rightarrow \infty} h(\tau)$. The scalar product on $\mathbb{H}_{i}$ is defined by

$$
\left\langle h_{1}, h_{2}\right\rangle_{\mathbb{H}_{i}}=h_{1}(\infty) h_{2}(\infty)+\sum_{j=1}^{i} \int_{(0, \infty)} h_{1}^{(j)}(\tau) h_{2}^{(j)}(\tau)(1+\tau)^{\gamma_{i}} d \tau
$$

For each $\zeta>0$ and $k, i \in \mathbb{N}_{0}$, we define the space $\mathbb{B}_{k}^{\zeta}\left(\mathbb{H}_{i} \times \mathbb{Y}\right)$ as the closure of $C_{b}^{k}\left(\mathbb{H}_{i} \times \mathbb{Y}\right)$ under the norm

$$
\|f\|_{\mathbb{B}_{h}^{\zeta}\left(\mathbb{H}_{i} \times \mathbb{Y}\right)}=\sum_{j=0}^{k} \sup _{(h, y) \in \mathbb{H}_{i} \times \mathbb{Y}}\left(\cosh \left(\zeta\|h\|_{\mathbb{H}_{i}}\right)+\|y\|_{\mathbb{Y}}^{2}\right)^{-1}\left\|D^{j} f(h, y)\right\|_{L\left(\left(\mathbb{H}_{i} \times \mathbb{Y}\right)^{j}\right)} .
$$

Together with Assumption 5.2 and 5.3, this implies that the conditions of 14, Sections 3.1.1 and 3.1.2] are satisfied for SPDE (5.5), 5.6 characterising the evolution of $(h, Y)$. (Note that $\beta_{y}$ needs to be bounded away from zero for $\mu_{y}^{\text {HJM }}$ and $\sigma_{y}^{\text {HJM }}$ to be bounded 
with bounded derivatives.) Thus, this SPDE admits unique solutions on each space $\mathbb{H}_{i} \times \mathbb{Y}$, given that the initial condition is smooth enough. The same applies to the SPDE for $h$ with fixed $y$ and the SDE for $Y$ with fixed $h$.

Fix $\zeta_{0}>\zeta>0$ and define $\mathbb{H}=\mathbb{H}_{2}, \mathbb{B}=\mathbb{B}_{0}^{\zeta_{0}}\left(\mathbb{H}_{2} \times \mathbb{Y}\right)$, and $\mathbb{B}^{\prime}=\mathbb{B}_{4}^{\zeta}\left(\mathbb{H}_{0} \times \mathbb{Y}\right)$. Then $(\mathcal{P}(t))_{t \geq 0},(\mathcal{Q}(t))_{t \geq 0}$, and $(\mathcal{R}(t))_{t \geq 0}$ are strongly continuous semigroups on $\mathbb{B}$ by 14 Lemma 13] and quasicontractive by [14, Lemma 7]. Their generators are denoted by $\mathcal{G}^{\mathcal{P}}, \mathcal{G}^{\mathcal{Q}}$, and $\mathcal{G}^{\mathcal{R}}$. By the same lemma, $\mathbb{B}^{\prime}$ is stable under $(\mathcal{P}(t))_{t \geq 0}$. Together with 14 , Theorem 11] this implies that for each $f \in \mathbb{B}^{\prime}$, the expressions

$$
\mathcal{G}^{\mathcal{P}} \mathcal{P}(t) f, \quad \mathcal{G}^{\mathcal{Q}} \mathcal{P}(t) f, \quad \mathcal{G}^{\mathcal{R}} \mathcal{P}(t) f, \quad \mathcal{G}^{\mathcal{Q}} \mathcal{G}^{\mathcal{Q}} \mathcal{P}(t) f, \quad \mathcal{G}^{\mathcal{Q}} \mathcal{G}^{\mathcal{R}} \mathcal{P}(t) f, \quad \mathcal{G}^{\mathcal{R}} \mathcal{G}^{\mathcal{Q}} \mathcal{P}(t) f
$$

are well-defined with $\mathbb{B}$-norm bounded uniformly in $t \in[0, T]$ and $\mathcal{G}^{\mathcal{P}} f=\mathcal{G}^{\mathcal{Q}} f+\mathcal{G}^{\mathcal{R}} f$. Thus, the splitting is of formal order one and the result follows from 20, Theorem 2.3 and Section 4.4].

5.7. Consistent recalibration property. If the coefficient $\beta$ in the HJM volatility $\sqrt{a} e^{\beta \tau}$ is stochastic, one would expect the forward rate process to reach every point in the Hilbert space with positive probability, i.e., the consistent recalibration property holds. This is made precise here.

Assumption 5.5. For each initial condition $Y(0)=y_{0} \in \mathbb{Y}$ and each $T>0$, the support of $Y_{T}$ is all of $\mathbb{Y}$. Moreover, $\left\{\beta_{y}: y \in \mathbb{Y}\right\}$ contains an interval $[\underline{\beta}, \infty)$ for some $\underline{\beta}$.

Assumption 5.6. The Hilbert space $\mathbb{H}$ is contained in $L_{\text {loc }}^{1}\left(\mathbb{R}_{+}\right)$, and each $h \in \mathbb{H}$ has a finite abscissa

$$
\operatorname{abs}(h)=\inf \left\{\beta \in \mathbb{R}: \int_{0}^{\infty} h(\tau) e^{\beta \tau} d \tau<\infty\right\}<\infty .
$$

The condition in Assumption 5.6 is mild; it is satisfied by the weighted Sobolev spaces in 16] and in particular by the space $\mathbb{H}$ of Theorem 5.4 . The above assumptions imply the consistent recalibration property, as the following theorem shows.

Theorem 5.7. The consistent recalibration property is satisfied for the Vasiček CRC model (5.5), 5.6) with respect to the state space $\mathcal{I}=\mathbb{H} \times \mathbb{Y}$.

Proof. Let $(h, Y)$ be the solution of (5.5) and (5.6) with initial value $\left(h_{0}, Y_{0}\right)$ and let $T \geq 0$. By 27, Theorem 1.1] the support of $\left(h_{T}, Y_{T}\right)$ is equal to the closure $\overline{\mathcal{L}_{T}}$ of $\mathcal{L}_{T}$, where $\mathcal{L}_{T}$ is the reachable set at time $T$ of the following control problem: in (5.5) and (5.6) the Brownian motions are replaced by piecewise continuously differentiable control functions. Let $(\hat{h}, \hat{Y})$ be the solution of (5.5) and (5.6) for the zero control. Taking variations in the control for $\hat{Y}$ implies that $\left\{\hat{h}_{T}\right\} \times \mathbb{Y} \subseteq \overline{\mathcal{L}_{T}}$ thanks to Assumption 5.5. Adding variations in the control of $h$ improves this to $\left(\left\{\hat{h}_{T}\right\}+\operatorname{span}\left\{\sigma_{y}^{\mathrm{HJM}}: y \in \mathbb{Y}\right\}\right) \times \mathbb{Y} \subseteq \overline{\mathcal{L}_{T}}$. The set $\operatorname{span}\left\{\sigma_{y}^{\mathrm{HJM}}: y \in \mathbb{Y}\right\}$ is dense in $\mathbb{H}$ because its orthogonal complement vanishes by [2. Proposition 1.7.2] and Assumption 5.6. Therefore, $\mathbb{H} \times \mathbb{Y} \subseteq \overline{\mathcal{L}_{T}}$, and the consistent recalibration property holds.

5.8. Example. We present an example of a Vasiček CRC model based on 10. In this model, the volatility is stochastic, but the speed of mean reversion is not. Therefore, the conditions of Theorem 5.7 are not satisfied, and it turns out that the model admits a finite-dimensional realisation. The explicit formula for bond prices in this model will serve as a reference for showing convergence of the numerical simulation scheme for CRC models in the continuous-time limit. 
The parameter process $Y$ is a CIR process with values in $\mathbb{Y}=\mathbb{R}_{+}$given by SDE 5.6 with a possibly correlated Brownian motion $\widetilde{W}$ and coefficients $\mu(y)=m+\mu y$ and $\sigma(y)=\sigma y$ for some $m \geq 0, \mu \leq 0$, and $\sigma \geq 0$. The Vasiček drift and volatility in the HJM equation are given by Equation (5.3) with $\beta_{y}=\beta$ for some constant $\beta<0$ and $a_{y}=y$, i.e.,

$$
\mu_{y}^{\mathrm{HJM}}(\tau)=-\frac{y}{\beta} e^{\beta \tau}\left(1-e^{\beta \tau}\right), \quad \sigma_{y}^{\mathrm{HJM}}(\tau)=\sqrt{y} e^{\beta \tau}, \quad \text { for all } \tau \in \mathbb{R}_{+} .
$$

If $h(0) \in C^{1}\left(\mathbb{R}_{+}\right)$, there is a closed-form solution of CRC equation (5.5),

$$
h(t, \tau)=h(0, t+\tau)-\int_{0}^{t} \frac{Y(s)}{\beta} e^{\beta(\tau+t-s)}\left(1-e^{\beta(\tau+t-s)}\right) d s+\int_{0}^{t} \sqrt{Y(s)} e^{\beta(\tau+t-s)} d W(s) .
$$

Setting $\xi(t)=\int_{0}^{t} Y(s) e^{2 \beta(t-s)} d s$ and $r(t)=h(t, 0)$, this can be rewritten as

$$
h(t, \tau)=h(0, t+\tau)+e^{\beta \tau}(r(t)-h(0, t))+\frac{1}{\beta}\left(e^{2 \beta \tau}-e^{\beta \tau}\right) \xi(t) .
$$

Setting $\tau=0$ in Equation (5.5) and plugging in Equation (5.7) yields

$$
d r(t)=(\mathcal{A} h(0, t)-\beta h(0, t)+\beta r(t)+\xi(t)) d t+\sqrt{Y(t)} d W(t) .
$$

Summing up, the process $X=(r, \xi, Y)$ is given by the SDE

$$
\left\{\begin{aligned}
d r(t) & =\left(\frac{\partial h}{\partial \tau}(0, t)-\beta h(0, t)+\beta r(t)+\xi(t)\right) d t+\sqrt{Y(t)} d W(t), \\
d \xi(t) & =(2 \beta \xi(t)+Y(t)) d t, \\
d Y(t) & =(m+\mu Y(t)) d t+\sigma \sqrt{Y(t)} d \widetilde{W}(t),
\end{aligned}\right.
$$

where $h(0) \in C^{1}\left(\mathbb{R}_{+}\right)$is a given initial forward rate curve. It follows that $X$ is an affine factor process for the short rate as described in Section 3 with $d=3, \ell=0, \lambda=(1,0,0)^{\top}$. Thus, the CRC model has a finite-dimensional realisation. If $\sigma=0$, the affine bond pricing formula is particularly simple: bond prices are given by

$$
P(t, T)=e^{\int_{t}^{T}\left(e^{\beta(s-t)} h(0, t)-h(0, s)\right) d s+\beta^{-1}\left(1-e^{\beta t}\right) r(t)-\frac{1}{2} \beta^{-2}\left(1-e^{\beta t}\right)^{2} \xi(t),}
$$

where $\xi(t)$ is deterministic and satisfies

$$
\xi(t)= \begin{cases}Y(0) \frac{e^{2 \beta t}-e^{\mu t}}{2 \beta-\mu}+\frac{m\left(2 \beta-\mu-2 \beta e^{\mu t}+\mu e^{2 \beta t}\right)}{2 \beta \mu(2 \beta-\mu)}, & \text { if } \mu<0, \\ Y(0) \frac{e^{2 \beta t}-1}{2 \beta}+\frac{m\left(e^{2 \beta t}-2 \beta t-1\right)}{4 \beta^{2}}, & \text { if } \mu=0,\end{cases}
$$

and $r(t)$ is normally distributed with mean

$$
e^{\beta t} r(0)+\int_{0}^{t} e^{\beta(t-s)}(\mathcal{A} h(0, s)-\beta h(0, s)+\xi(s)) d s,
$$

and variance

$$
\frac{Y(0)}{2 \beta}\left(e^{2 \beta t}-1\right)+\frac{m}{4 \beta^{2}}\left(-2 \beta t+e^{2 \beta t}-1\right) .
$$


5.9. Calibration of Vasiček CRC models. As outlined in Section 4.12, we first consider $y$ as fixed and suppress the dependence on $y$ in the notation. For any selection of times to maturity $\tau_{i}, \tau_{j}$, estimators for $a, \beta$ can be obtained as described in Section 3.9 by solving for those $\widehat{a}, \widehat{\beta}$ which achieve the best fit in (3.13), i.e.,

$$
\begin{aligned}
\frac{\left[\widehat{r}\left(\cdot, \tau_{i}\right), \widehat{r}\left(\cdot, \tau_{j}\right)\right]\left(t_{n}\right)-\left[\widehat{r}\left(\cdot, \tau_{i}\right), \widehat{r}\left(\cdot, \tau_{j}\right)\right]\left(t_{n-M}\right)}{t_{n}-t_{n-M}} & \approx a \frac{e^{\beta \tau_{i}}-1}{\beta \tau_{i}} \frac{e^{\beta \tau_{j}}-1}{\beta \tau_{j}} \\
& \approx a \frac{\beta \tau_{i}+\beta^{2} \tau_{i}^{2} / 2}{\beta \tau_{i}} \frac{\beta \tau_{j}+\beta^{2} \tau_{j}^{2} / 2}{\beta \tau_{j}} .
\end{aligned}
$$

Varying the calibration time $t_{n}$ creates a time series of coefficients $\widehat{a}\left(t_{n}\right), \widehat{\beta}\left(t_{n}\right)$ for which we need to specify and calibrate a model. Some models are described in Section 7.5. below.

\section{Consistent Recalibration of Cox-Ingersoll-Ross models}

6.1. Overview. We give a brief overview of CRC models based on CIR short rates. The overview is sufficient to set the notation for the empirics in Section 7. A detailed description is provided in the online appendix to this paper. For comparison, we briefly digress to the CIR ++ model and its CRC version.

6.2. Hull-White extended Cox-Ingersoll-Ross models. We use the setup of Sections 3.2 and 4.2 setting $\mathbb{X}=\mathbb{R}_{+}, \ell=0, \lambda=1$. We do not specify the parameter space $\mathbb{Y}$, yet, but we assume that for each $(x, y) \in \mathbb{X} \times \mathbb{Y}$, the volatility and drift coefficients are given by $A_{y}(x)=\alpha_{y} x$ and $B_{y}(x)=\beta_{y} x$ for some $\alpha_{y} \in(0, \infty)$ and $\beta_{y} \in(-\infty, 0)$. For simplicity, we again choose equidistant grids of times $t_{n}=n \delta$ and times to maturity $\tau_{n}=n \delta$, for all $n \in \mathbb{N}_{0}$, where $\delta$ is a positive constant.

The CRC algorithm is similar to the Vasiček model, with the following important differences:

- The admissibility condition in Assumption 3.1 is satisfied if and only if $\theta(t) \geq 0$, for all $t \in \mathbb{R}_{+}$. This condition can be problematic in practise, as discussed in Section 7.4. Moreover, if this condition is expressed in terms of forward rates $h(t)=$ $\mathcal{H}_{y}(\theta(t), X(t))$ instead of Hull-White extensions $\theta(t)$, it becomes apparent that the set of admissible forward rate curves depends on the parameter $y$. This makes it difficult to formulate convergence results similar to those in the Vasiček case.

- In contrast to the Vasiček model, there does not seem to be a closed-form expression for $\theta=\mathcal{C}_{y}(h, x)$ because the Volterra kernel in the integral operator $\mathcal{H}_{y}$ is more complicated. Therefore, the Volterra equation is solved by numerical approximation of order two using a discretisation in the time to maturity.

6.3. CIR++ models in the CRC framework. In the CIR ++ model 7, Section 3.9], also known as deterministic shift-extended CIR model, the short rate process is defined by $r(t)=X(t)+\theta(t)$, where $X$ is a CIR process and $\theta$ is a deterministic function of time. Note that this is a different time-inhomogeneity than the one described in Section 6.2. In particular, the factor process $X$ is time-homogeneous and does not coincide with the short rate. The HJM equation of the CIR ++ model is

$$
\begin{aligned}
d h(t) & =\left(\mathcal{A} h(t)+\mu_{y}^{\mathrm{HJM}}(X(t))\right) d t+\sigma_{y}^{\mathrm{HJM}}(X(t)) d W(t), \\
d X(t) & =\left(b_{y}+\beta_{y} X(t)\right) d t+\sqrt{\alpha_{y} X(t)} d W(t),
\end{aligned}
$$


where $\mu_{y}^{\mathrm{HJM}}$ and $\sigma_{y}^{\mathrm{HJM}}$ are the same as in the CIR case. In the CRC extension of this model, $y$ is replaced by a stochastic process $Y$. This model has both advantages and disadvantages over the consistently recalibrated CIR model:

- The SDE for $X$ does not depend on $h$. Therefore, existence and uniqueness of $X$ can be shown by standard methods. Then a mild solution $h$ can be constructed by stochastic convolution [13, Section 6.1]:

$$
h(t)=\mathcal{S}(t) h(0)+\int_{0}^{t} \mathcal{S}_{t-s} \mu_{Y(s)}^{\mathrm{HJM}}(X(s)) d s+\int_{0}^{t} \mathcal{S}_{t-s} \sigma_{Y(s)}^{\mathrm{HJM}}(X(s)) d W(s) .
$$

- The function $\theta$ is allowed to assume negative values and can be calibrated to a given yield curve without having to invert a Volterra integral operator. However, this calibration requires one to know the current value of $X$. This can be seen from the equation for forward rate curves

$$
h(t)=\mathcal{S}(t) \theta-b_{y} \Psi_{y}-\Psi_{y}^{\prime} X(t),
$$

where $\Psi_{y}$ is the same as in the CIR case (c.f. Section 3.4). In contrast to the CIR model, the process $X$ is not directly observable from the short end of the term structure. Therefore, $X(t)$ and the parameters $\alpha_{y}$ and $\beta_{y}$ have to be estimated jointly from realised covariations of yields as described in Section 3.7. Moreover, in contrast to the CIR model the parameter $b_{y}$ is not redundant and has to be estimated using the same methodology as for general multi-factor models (see Section 3.7).

\section{EMPIRICAL RESULTS}

7.1. Overview. CRC models based on Vasiček and CIR short rates are calibrated to Euro area yield curves. Properties of the calibrated models are studied in comparison to market data and models without consistent recalibration. Our empirical findings show that the assumption of constant parameters in the Vasiček and CIR models is too restrictive. Therefore, the additional flexibility provided by CRC models is a useful tool to better capture the market dynamics. This is also reflected in better fits of the covariance matrix of yields.

7.2. Description of the data. We consider the zero-coupon yield curves released by the European Central Bank (ECB) on a daily basis. The yields are estimated from AAA-rated (Fitch Ratings) Euro area central government bonds being actively traded on the market. Estimation is done by the ECB using the Svensson family of curves, see [31, 33. Data is available from September 6, 2004, and we set April 1, 2014 to be the last observation date. In total, this results in 2454 observed yield curves with times to maturity ranging from 3 months up to 30 years. Yields are continuously compounded (c.f. Equation (3.11) and denoted by $\widehat{r}(t, \tau)$, with $\tau$ being the time to maturity. A selection of yields is shown in Figures 7.1 and 7.2 . The short rate is approximated by the yield with the lowest time to maturity (3 months) and is depicted in Figure 7.1

7.3. Calibration of CRC models. The CRC models based on Vasiček and CIR short rates are calibrated as described in Section 5.9 and the online appendix. Time steps $\delta=240^{-1}$ of one business day and time windows of $M=100$ business days are used for the calibration. The choice $M=100$ is a compromise between accuracy and over-smoothing and gives reasonable results over the time horizon of roughly a decade (see Figures 7.3 and 


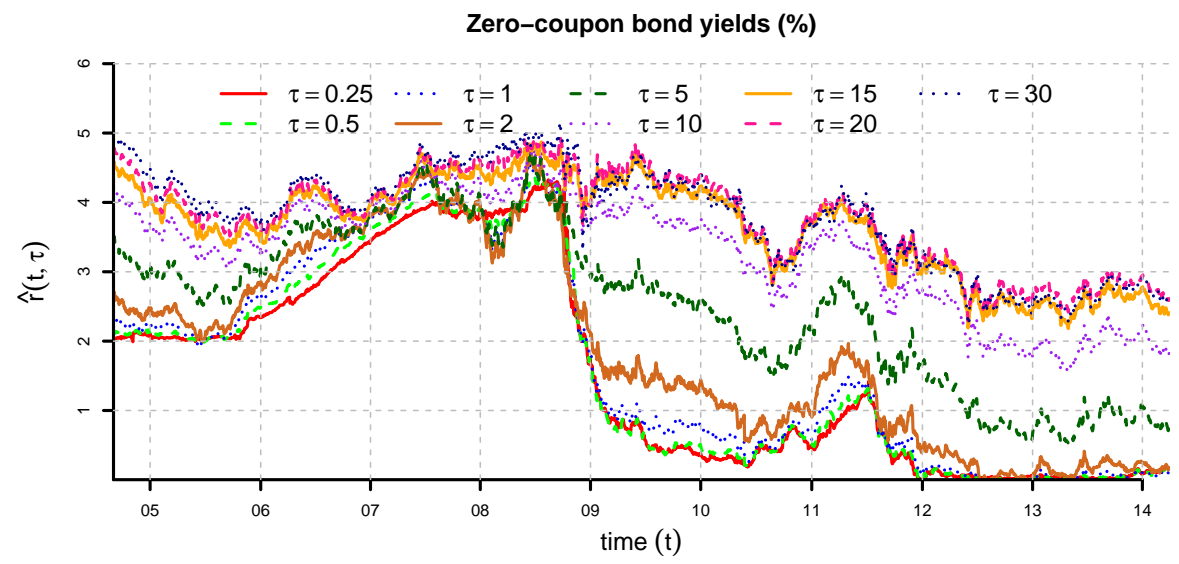

Figure 7.1. Historical zero-coupon yields estimated by the ECB for various times to maturity from $06 / 09 / 2004$ to $01 / 04 / 2014$. We use the 3 -month yields $(\tau=0.25)$ as a proxy for the short rate.

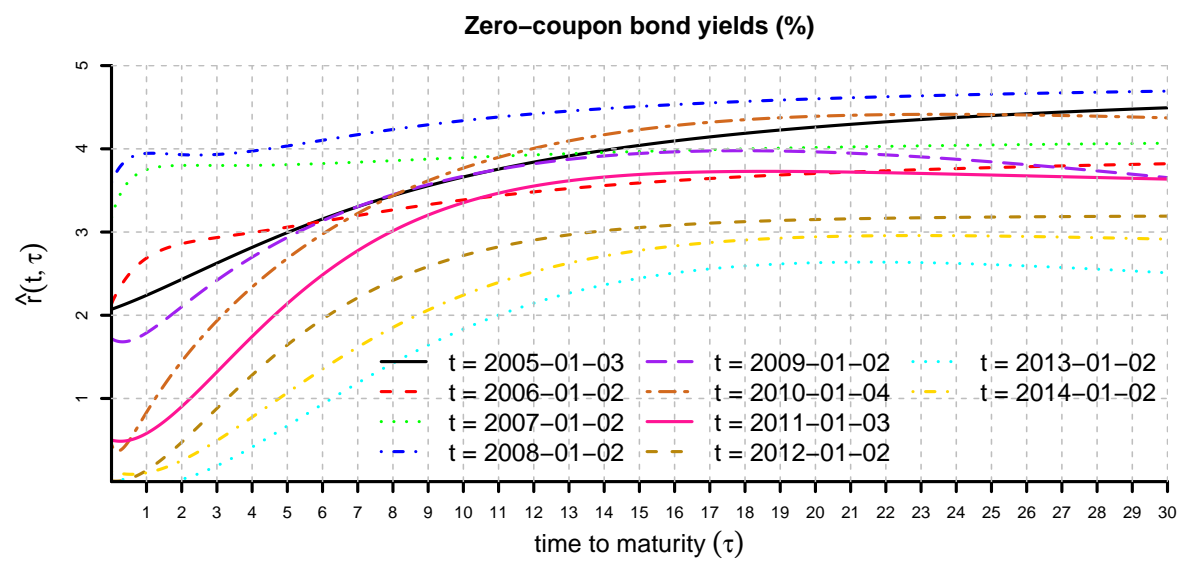

Figure 7.2. Zero-coupon yield curves estimated by the ECB for various observation dates.

7.4). For $\tau_{1} \ll 1$ one immediately obtains from Equation (5.11) and its CIR counterpart the estimator

$$
\hat{a}(t)=\frac{\left[\widehat{r}\left(\cdot, \tau_{1}\right), \widehat{r}\left(\cdot, \tau_{1}\right)\right](t)-\left[\widehat{r}\left(\cdot, \tau_{1}\right), \widehat{r}\left(\cdot, \tau_{1}\right)\right](t-\delta M)}{\delta M},
$$

in the Vasiček case, and

$$
\hat{\alpha}(t)=\frac{\left[\widehat{r}\left(\cdot, \tau_{1}\right), \widehat{r}\left(\cdot, \tau_{1}\right)\right](t)-\left[\widehat{r}\left(\cdot, \tau_{1}\right), \widehat{r}\left(\cdot, \tau_{1}\right)\right](t-\delta M)}{\delta \sum_{m=0}^{M-1} \hat{r}\left(t-\delta m, \tau_{1}\right)},
$$




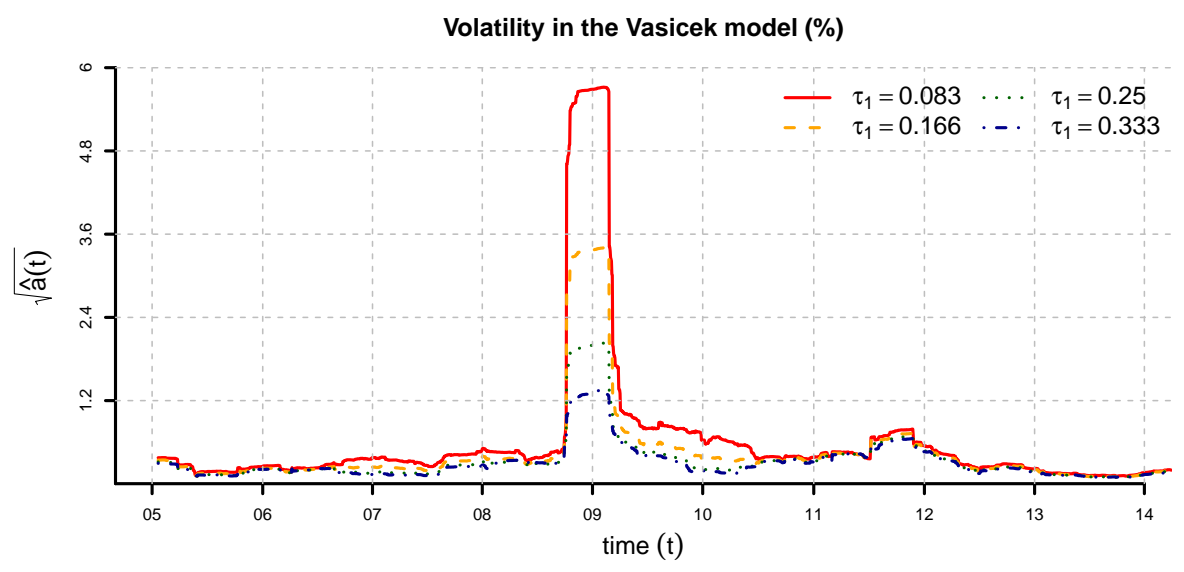

FiguRE 7.3. Volatility parameter of the Vasiček model estimated by (7.1) using a time window of $M=100$ yields with time to maturity $\tau_{1}$.

in the CIR case, where the quadratic variation is estimated by (5.11). On the other hand, taking $\tau_{2} \gg 1$, one can solve (5.11) and its CIR counterpart for $\beta$ and obtain the estimator

$$
\hat{\beta}(t)=-\frac{1}{\tau_{2}}\left(\frac{\delta M \hat{a}(t)}{\left[\widehat{r}\left(\cdot, \tau_{2}\right), \widehat{r}\left(\cdot, \tau_{2}\right)\right](t)-\left[\widehat{r}\left(\cdot, \tau_{2}\right), \widehat{r}\left(\cdot, \tau_{2}\right)\right](t-\delta M)}\right)^{\frac{1}{2}},
$$

in the Vasicek case, and

$$
\begin{aligned}
\hat{\beta}(t)= & \frac{\sqrt{\hat{\alpha}(t)}}{2} \tau_{2}\left(\frac{\left[\widehat{r}\left(\cdot, \tau_{2}\right), \widehat{r}\left(\cdot, \tau_{2}\right)\right](t)-\left[\widehat{r}\left(\cdot, \tau_{2}\right), \widehat{r}\left(\cdot, \tau_{2}\right)\right](t-\delta M)}{\delta \sum_{m=0}^{M-1} \hat{r}\left(t-\delta m, \tau_{1}\right)}\right)^{\frac{1}{2}} \\
& -\frac{\sqrt{\hat{\alpha}(t)}}{\tau_{2}}\left(\frac{\left[\widehat{r}\left(\cdot, \tau_{2}\right), \widehat{r}\left(\cdot, \tau_{2}\right)\right](t)-\left[\widehat{r}\left(\cdot, \tau_{2}\right), \widehat{r}\left(\cdot, \tau_{2}\right)\right](t-\delta M)}{\delta \sum_{m=0}^{M-1} \hat{r}\left(t-\delta m, \tau_{1}\right)}\right)^{-\frac{1}{2}},
\end{aligned}
$$

in the CIR case. The resulting trajectories of the estimated Vasiček volatility $\sqrt{a}$ and CIR volatility $\sqrt{\alpha}$ are shown in Figures 7.3 and 7.4 . The trajectories of the speed of mean reversion $-\beta$ for both models are plotted in Figures 7.5 and 7.6

It turns out that for most parts of the data, $a$ and $\alpha$ do not depend too much on the times to maturity used in the estimation, whereas $\beta$ does. Typically, smaller times to maturity result in larger values of $-\beta$, as shown in Figures 7.5 and 7.6 . This means that one-factor Vasiček and CIR models are not flexible enough to reproduce the ECB yield curve movements in full accuracy, and so the choice of times to maturity used in the calibration procedure may have an impact on the results. In particular we set $\tau_{1}=0.25$ and $\tau_{2}=2$ (i.e. 3 months and 2 years).

The dependence of the estimated parameter $\beta$ on the choice of times to maturity suggests to use multi-factor models as building blocks for CRC models. In the empirical part of this paper, we aim for a detailed understanding of the one-factor case and leave the extension to multiple factors for future research. As pointed out, accurate modelling of long-term rates might require one to enlarge the model class even further to allow the forward rate volatilities to decay slower than exponentially. 


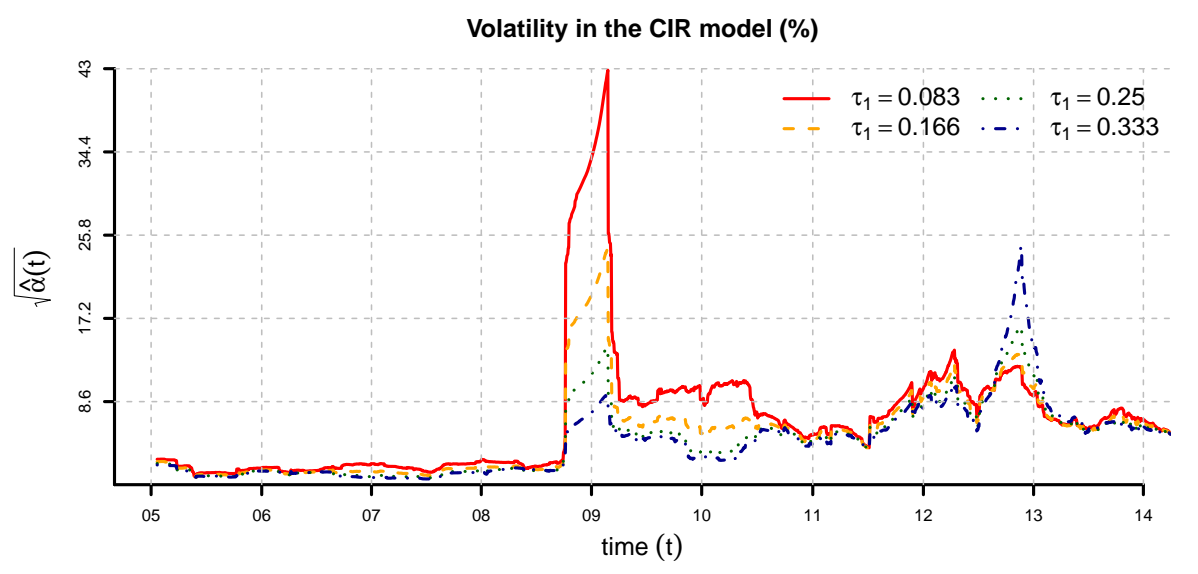

FiguRE 7.4. Volatility parameter of the CIR model estimated by 7.2 using a time window of $M=100$ yields with time to maturity $\tau_{1}$.

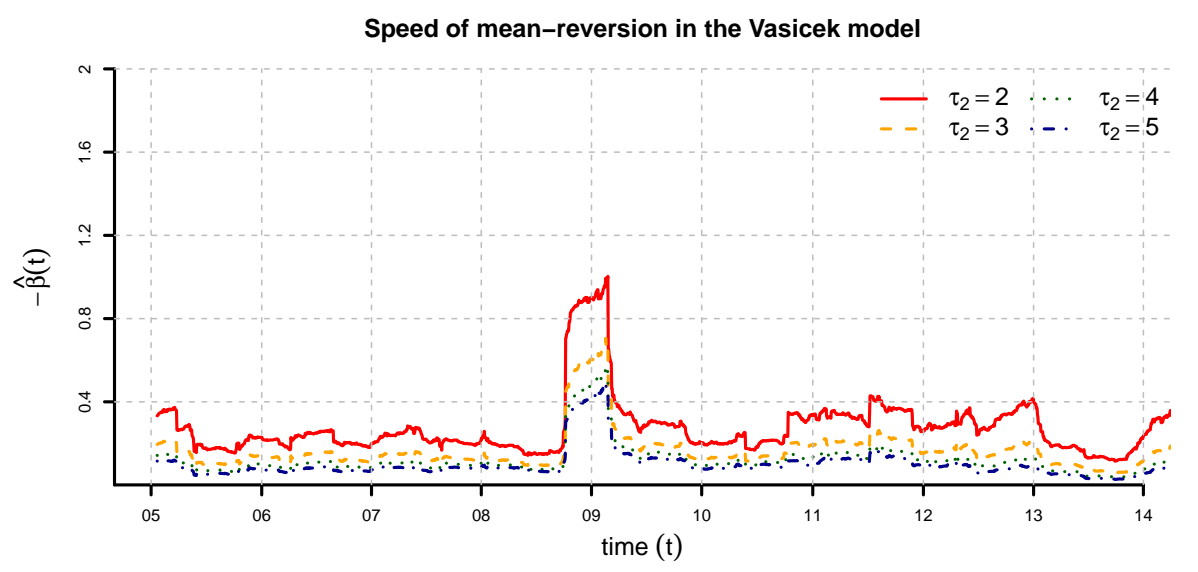

FiguRE 7.5. Speed of mean-reversion parameter of the Vasiček model estimated by (7.3) using a time window of $M=100$ yields with times to maturity $\tau_{1}=0.25$ and various values of $\tau_{2}$.

By the theory of Hull-White extensions, an exact match to the initial yield curve is always achieved, but the corresponding time-homogeneous models often do not match the initial yield curve well (Figures 7.7 and 7.8). This is not surprising as they are calibrated to the yield curve dynamics and not to the initial yield curve. This separation of dynamics and initial calibration is actually one of the strengths of our approach.

To model the dynamics of the Vasiček coefficients $a, \beta$ and the CIR coefficients $\alpha$, $\beta$, we use geometric Brownian motions and/or CIR processes, as laid out in Section 7.5. Note that the assumption of constant coefficients, which is implicit in affine factor models without the CRC extension, is not realistic over long time horizons in view of Figures 7.3 and 7.4 


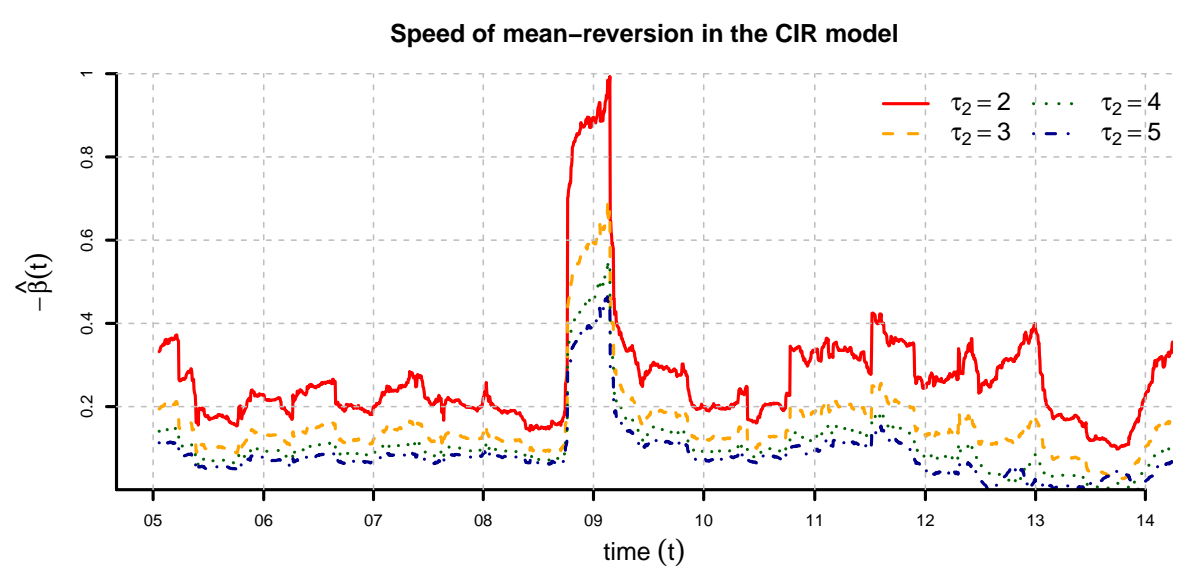

FiguRE 7.6. Speed of mean-reversion parameter of the CIR model estimated by (7.4) using a time window of $M=100$ yields with times to maturity $\tau_{1}=0.25$ and various values of $\tau_{2}$.

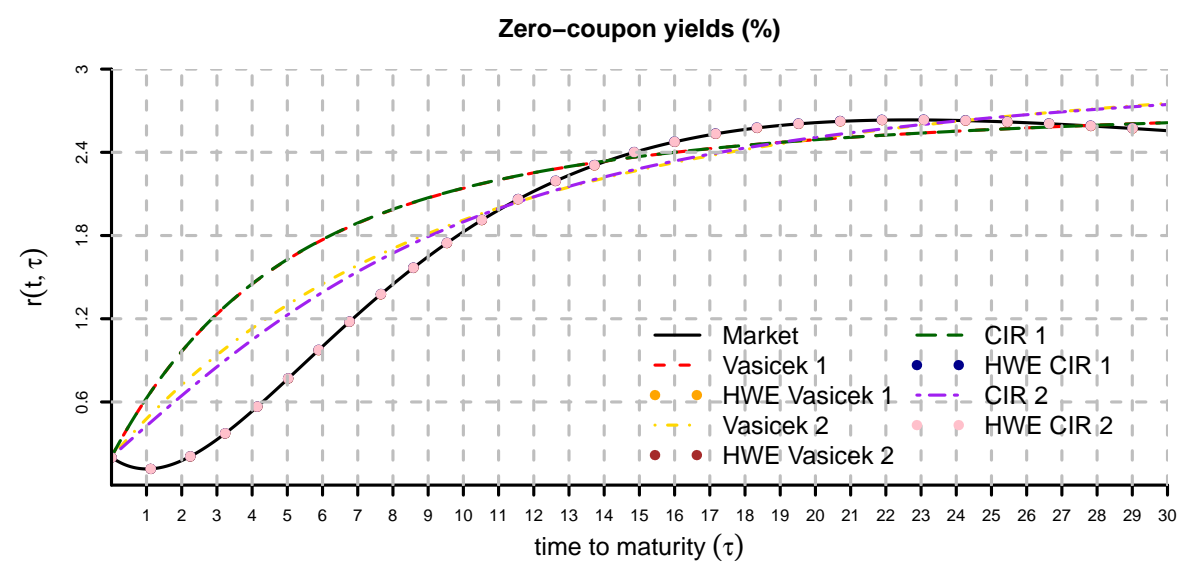

FiguRE 7.7. Calibrations of some homogeneous and Hull-White extended models as of 1 April 2014. Vasicek 1 and CIR 1 are homogeneous models calibrated to the yield curve dynamics using (7.1)- 7.4 with $\tau_{1}=0.25$ and $\tau_{2}=2$. Vasiček 2 and CIR 2 are homogeneous models calibrated to the prevailing yield curve by least squares. The Hull-White extended models match the initial yield curve exactly.

7.4. Negative levels of mean reversion. A problematic aspect is that the timedependent drift $\theta$ obtained by the calibration to the initial yield curve can assume negative values, which are not admissible in the CIR model. The problem occurs mostly in low interest rate scenarios with partially inverted yield curves (Figures 7.7, 7.8, and 7.9. 


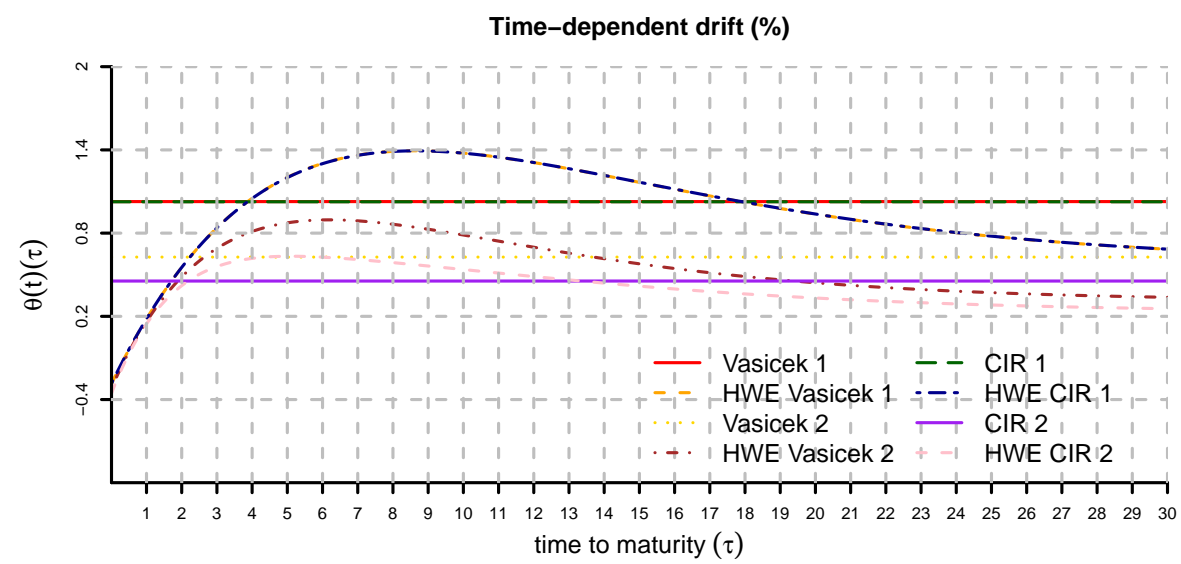

Figure 7.8. The Hull-White extensions $\theta(t)$ corresponding to the models of Figure 7.7. Note that $\theta(t)$ assumes negative values, which is typical in situations where the yield curve is inverted at the short end.

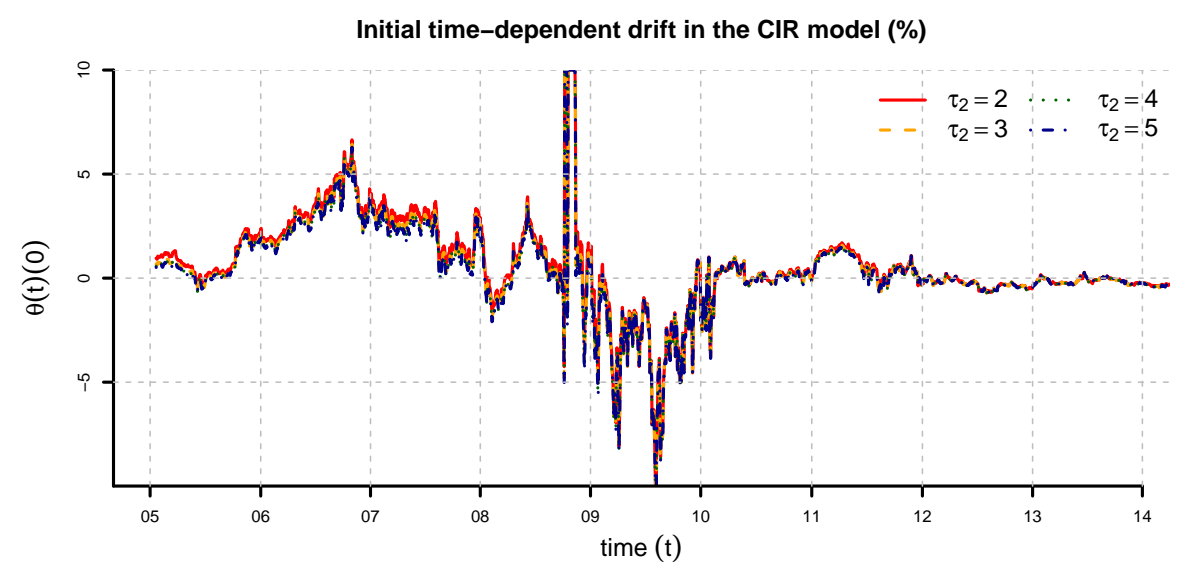

FIGURE 7.9. The historical values of $\theta(t)(0)$ in the CIR model calculated using the estimates of $\beta$ in Figure 7.6. Negative values occur frequently in 2009 and 2012 to 2014. They are problematic for reasons laid out in Section 7.4

In contrast, negative values of $\theta$ are allowed in the Vasiček model and might even be desirable for modelling bond markets with negative interest rates.

As only the short (left) end of $\theta$ is relevant for CRC models, at each step of the simulation scheme (cf. Algorithm 5.1), it is sufficient to understand how $\theta(0)$ depends on the prevailing forward rate curve and the coefficients of the affine factor process. The 
general formula for $\theta(0)$ is

$$
\theta(0)=\mathcal{C}_{y}(h, x)(0)=\frac{1}{\left\langle\lambda, e_{1}\right\rangle}\left(h^{\prime}(0)-F_{y}^{\prime}(0) \cdot \lambda-\left\langle R_{y}^{\prime}(0) \cdot \lambda, x\right\rangle\right),
$$

which follows by differentiating the relation $h=\mathcal{H}_{y}(\theta, x)$ with respect to the time to maturity $\tau$ and evaluating at $\tau=0$. In the Vasiček and CIR case, the formula becomes $\theta(0)=h^{\prime}(0)-\beta_{y} h(0)$. This shows that the problem can be alleviated to some extent by artificially choosing higher levels of $-\beta$, resulting in higher values of $\theta(0)$. For this reason we set $\tau_{2}=2$ instead of higher values of $\tau_{2}$ in the calibration of $\beta$ (Figures 7.5 and 7.6). Despite this correction, $\theta(0)$ remains negative for most parts of 2009 and 2012-2014. During these periods of time, the CIR model cannot be fitted simultaneously to the yield curves as well as their volatilities and has to be rejected.

7.5. Models for parameter evolutions. There are very few restrictions on the choice of parameter process. It can be chosen exogenously for scenario based simulation or calibrated to the market, and it is not restricted by the fit to the initial term structure.

We consider here four models for the evolution of the Vasiček and CIR parameters: a reference model where the parameters are constant, two toy models with constant mean reversion and time-varying volatility, and one fully stochastic model which is calibrated to the market. In the Vasiček case, the four models are:

(V1) a Hull-White extended Vasiček model with constant coefficients $\beta_{y}=\beta_{0}$ and $a_{y}=a_{0}$;

(V2) a Vasiček CRC model with constant mean reversion coefficient $\beta_{y}=\beta_{0}$ and deterministically increasing volatility given by $a_{y}=a_{0} y, Y(t)=1+3 t$;

(V3) a Vasiček CRC model with constant mean reversion coefficient $\beta_{y}=\beta_{0}$ and stochastically increasing volatility given by $a_{y}=y, d Y(t)=\left(4 a_{0}-Y(t)\right) d t+\sigma \sqrt{Y(t)} d \widetilde{W}(t)$, $Y(0)=a_{0}, \sigma=3 \cdot 10^{-3} ;$ and

(V4) a Vasiček CRC model with stochastic coefficients given by geometric Brownian motion: $\beta_{y}=y_{1}, a_{y}=y_{2}, d Y_{1}=\mu_{1} Y_{1}(t)+\sigma_{1} Y_{1}(t) d \widetilde{W}_{1}(t), d Y_{2}=\mu_{2} Y_{2}(t)+$ $\sigma_{2} Y_{2}(t) d \widetilde{W}_{2}(t)$. The coefficients $\mu_{1,2}$ and $\sigma_{1,2}$ are deterministic and calibrated to $M$ observations as described in Section 7.3.

Note that models (V2) (V3) both describe scenarios where the standard deviation of the noise in the short rate process doubles over the period of a year. Indeed, in (V2) the volatility coefficient $a$ increases to four times its initial value, and in (V) the level of mean reversion of $a$ increases to four times its initial value.

Models (V2) and (V3) are special cases of Section 5.8. In (V2), which corresponds to $m=3 a_{0}, \mu=0$, and $\sigma=0$, there is an explicit formula for the moment generating function of the short rate process. By equations $(5.9)$ and $(5.10)$, it is given by

$$
\mathbb{E}\left[e^{\eta r(t)}\right]=e^{e^{\beta_{0} t} \eta r_{0}+\eta \int_{0}^{t} e^{\beta_{0}(t-s)}\left(h_{0}^{\prime}(s)-\beta h_{0}(s)+\xi(s)\right) d s+\frac{\eta^{2}}{2} \xi(t)}, \quad \eta \in \mathbb{R}, t \in \mathbb{R}_{+},
$$

where

$$
\xi(t)=a_{0} \frac{e^{2 \beta_{0} t}-1}{2 \beta_{0}}+\frac{3 a_{0}\left(e^{2 \beta_{0} t}-2 \beta_{0} t-1\right)}{4 \beta_{0}^{2}} .
$$

Model (V3) corresponds to Section 5.8 with $m=4 a_{0}, \mu=-1$ and $\sigma=3 \cdot 10^{-3}$.

The semigroup approach of Theorem 5.4 implies convergence of the simulation scheme for (V2) and for (V4 with $Y_{1}$ replaced by $Y_{1}+\epsilon$ for some $\epsilon>0$. In our numerical simulations, we observe convergence for all models (see Section 7.7), including the following CIR counterparts of the models just described: 
(CIR1) a Hull-White extended Cox-Ingersoll-Ross model with constant coefficients $\beta_{y}=\beta_{0}$ and $\alpha_{y}=\alpha_{0}$;

(CIR2) a Cox-Ingersoll-Ross CRC model with constant mean reversion coefficient $\beta_{y}=\beta_{0}$ and deterministically increasing volatility given by $\alpha_{y}=\alpha_{0} y, Y(t)=1+3 t$;

(CIR3) a Cox-Ingersoll-Ross CRC model with constant mean reversion coefficient $\beta_{y}=\beta_{0}$ and stochastically increasing volatility given by $\alpha_{y}=y, d Y(t)=\left(4 \alpha_{0}-Y(t)\right) d t+$ $\sigma \sqrt{Y(t)} d \widetilde{W}(t), Y(0)=\alpha_{0}, \sigma=5 \cdot 10^{-2} ;$ and

(CIR4) a Cox-Ingersoll-Ross CRC model with stochastic coefficients given by geometric Brownian motion: $\beta_{y}=y_{1}, \alpha_{y}=y_{2}, d Y_{1}=\mu_{1} Y_{1}(t)+\sigma_{1} Y_{1}(t) d \widetilde{W}_{1}(t)$, $d Y_{2}=\mu_{2} Y_{2}(t)+\sigma_{2} Y_{2}(t) d \widetilde{W}_{2}(t)$. The coefficients $\mu_{1,2}$ and $\sigma_{1,2}$ are deterministic and calibrated to $M$ observations as described in Section 7.3.

7.6. Implementation. Simulating CRC models requires iterative sampling of the underlying affine short rate process and recalibrating Hull-White extensions. This is explained in detail in Algorithm 5.1 for the Vasiček model and in the online appendix for the CIR model. The algorithms can be parallelised on a path-by-path level. Parallelisation on lower levels does not pay off because the individual time steps are dependent on each other. In our implementation in $\mathrm{R}$, generating $10^{5}$ paths with 240 time steps on a cluster of 48 times $2.2 \mathrm{GHz}$ processors takes around 10 minutes in the Vasiček case and 20 minutes in the CIR case.

7.7. Convergence analysis. Theorem 5.4 predicts first order convergence of the simulation scheme under suitable assumptions on the model. The objective of this section is to demonstrate this convergence in numerical examples for the models described in Section 7.5.

The simulation is started with the initial forward rate curve $h_{0}$ of September 2, 2013. The parameters $\beta_{0}, a_{0}, \alpha_{0}, \theta_{0}$ are calibrated as in Section 7.3 with a time window of $M=100$ observations. Then the moment generating function of the short rate $r(1)$ after one year is calculated by Monte Carlo simulation with $10^{5}$ sample paths. In the model (V2), the exact value of the moment generating function is known and given by Equation (7.5) In the other models, a reference value is calculated by extrapolation from the Monte Carlo estimates. The resulting errors are shown in Figures 7.10, 7.11, and 7.12 As expected from Theorem 5.4 we observe first order convergence for models (V2) and (V4). The errors in Figure 7.12 indicate convergence also in the CIR counterparts.

7.8. Distributional properties. Making parameters in the CIR and Vasiček model stochastic in the sense of CRC models has considerable impact on the distribution of short rates and prices. As an example, statistics of the short rate $r(1)$ obtained by simulation are presented in Table 7.1. In the models (V1) and (V2) with deterministic parameters, the short rate process is Gaussian (see Section 5.8). As expected, the simulations show skewness and excess kurtosis values close to zero. In contrast, leptokurtosis appears in the models ( $\mathrm{V} 3$ and $(\sqrt{4})$ with stochastic parameters. In the CIR examples, the distribution of $r(1)$ is also affected considerably by the stochasticity of the parameters.

7.9. Covariation of yields. A further example where empirical differences between $\mathrm{CRC}$ and non-CRC models become apparent is the covariation matrix of yields. By the Dubins-Schwarz theorem, the covariation process determines the distribution of yields and therefore the prices of bond options. On short time intervals, the covariations are closely related to covariances, which are a key determinant of the prices of call and put options.

In the Hull-White Vasiček and CIR models without the CRC extension, the covariation matrix of yields with different times to maturity has rank 1 . This is in stark contrast to 


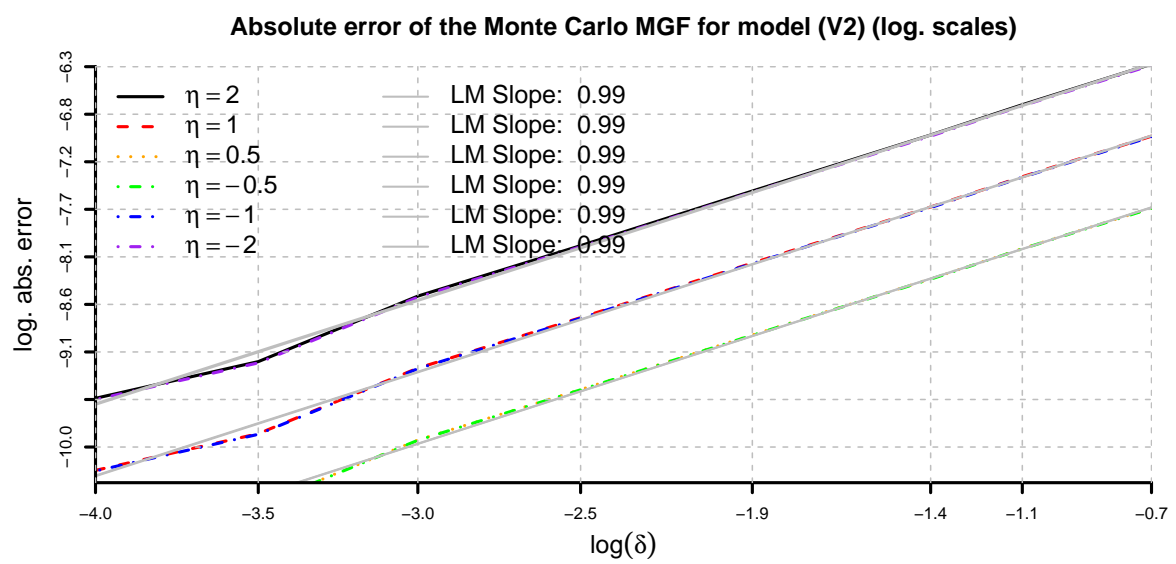

Figure 7.10. Absolute error (log-log plot) of the Monte Carlo estimate of the moment generating function $E\left[e^{\eta r(1)}\right]$ for model (V2). This is calculated as the absolute difference between the estimate and 7.5 for different values of $\delta$. We simulate $10^{5}$ paths for the estimation.

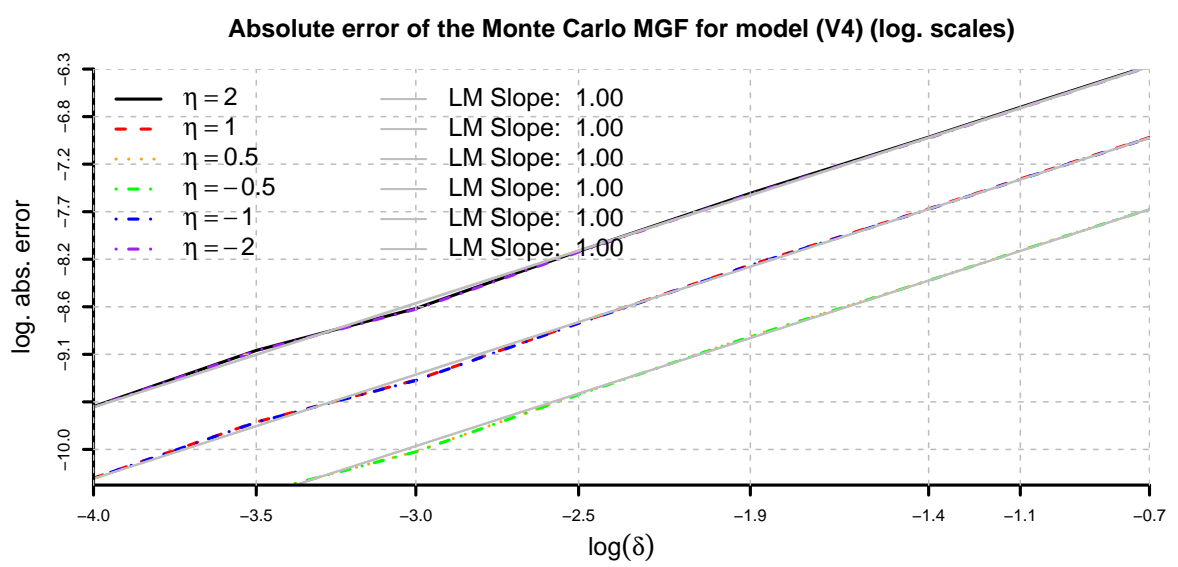

Figure 7.11. Absolute error (log-log plot) of the Monte Carlo estimate of the moment generating function $E\left[e^{\eta r(1)}\right]$ for model ( $\sqrt{4}$ ) defined in Section 7.5. The true values are estimated by the intercept of the linear extrapolation of the Monte Carlo estimates. The errors are calculated as the absolute difference between the intercept and the estimates. $10^{5}$ paths were used in the simulation.

the covariations observed in the market. For instance, the $33 \times 33$ covariation matrix of market yields with times to maturity ranging from 3 months to 30 years typically has rank between 5 and 9, as shown in Figure 7.13. The ranks produced by the CRC models 


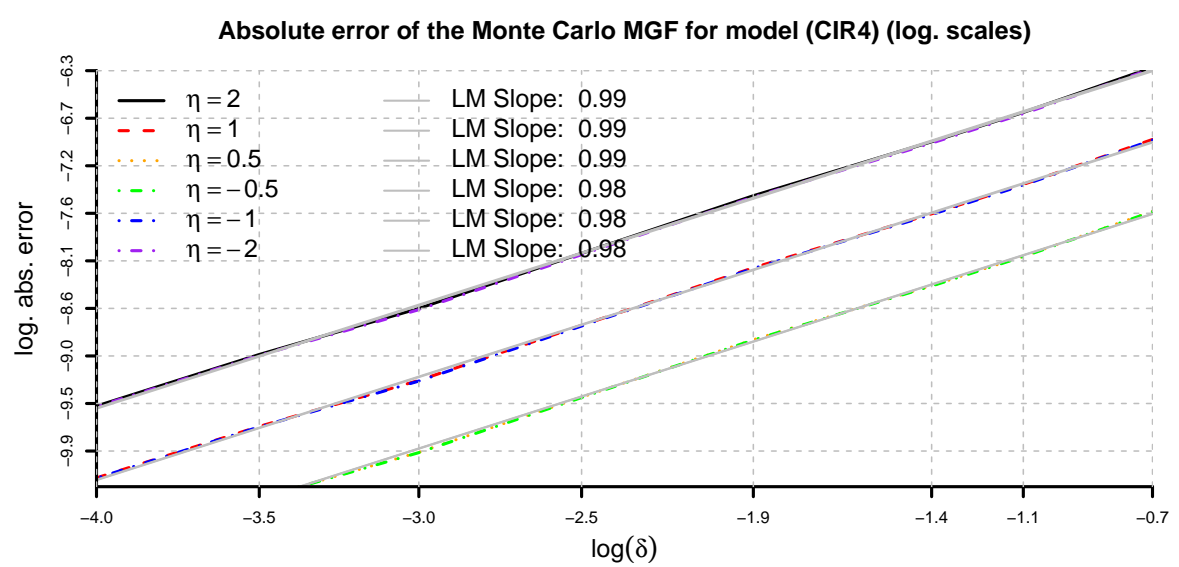

Figure 7.12. Absolute error (log-log plot) of the Monte Carlo estimate of the moment generating function $E\left[e^{\eta r(1)}\right]$ for model (CIR 4 ). The true values are estimated by the intercept of the linear extrapolation of the Monte Carlo estimates. The errors are calculated as the absolute difference between the intercept and the estimates for different values of $\delta .10^{5}$ paths were used in the simulation.

\begin{tabular}{|c|c|c|c|c|c|c|c|c|}
\hline & (V1) & (V2) & (V3) & (V4) & (CIR1) & (CIR2) & (CIR3) & (CIR4) \\
\hline Mean (\%) & 0.25 & 0.24 & 0.25 & 0.24 & 0.25 & 0.25 & 0.25 & 0.24 \\
\hline Median (\%) & 0.25 & 0.24 & 0.25 & 0.24 & 0.20 & 0.12 & 0.14 & 0.19 \\
\hline Volatility (\%) & 0.09 & 0.15 & 0.13 & 0.07 & 0.19 & 0.33 & 0.30 & 0.20 \\
\hline Skewness & -0.02 & 0.00 & 0.02 & 0.00 & 1.79 & 2.75 & 2.63 & 1.90 \\
\hline Kurtosis & 3.01 & 2.99 & 4.94 & 3.21 & 8.24 & 14.47 & 13.61 & 9.06 \\
\hline Minimum (\%) & -0.19 & -0.36 & -0.82 & -0.22 & 0.00 & 0.00 & 0.00 & 0.00 \\
\hline Maximum (\%) & 0.60 & 0.82 & 1.22 & 0.61 & 2.06 & 4.61 & 3.95 & 2.21 \\
\hline 1st Quartile (\%) & 0.18 & 0.14 & 0.17 & 0.20 & 0.11 & 0.04 & 0.05 & 0.10 \\
\hline 3rd Quartile (\%) & 0.31 & 0.34 & 0.32 & 0.29 & 0.33 & 0.33 & 0.33 & 0.33 \\
\hline
\end{tabular}

TABLE 7.1. Statistics of the short rate $r(1)$ at time 1 in the models defined in Section 7.5 obtained by Monte-Carlo simulations. $10^{5}$ paths and a step size of $\delta=0.02$ were used in the simulation.

(V4) and (CIR 44) typically lie between 3 and 5. Thus, they are higher than those of the non-CRC models, but not as high as those of the market.

Numerically, the covariation matrix is calculated as in (5.11) and the ranks are defined as the number of singular values differing significantly from zero. A comparison with Figures 7.15 and 7.14 shows that higher ranks are also related to higher volatility of the parameters. 


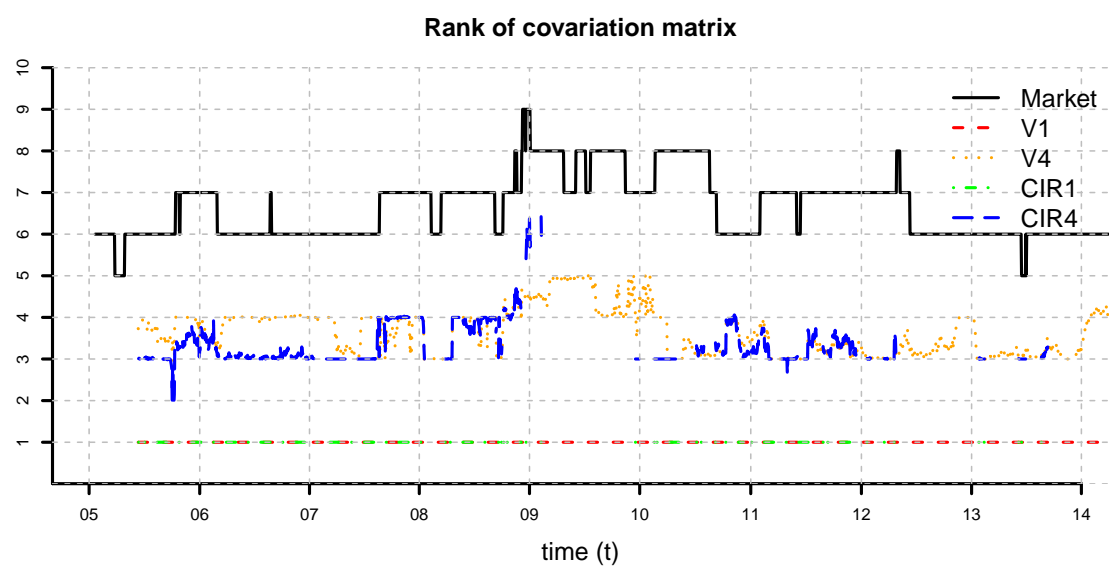

FiguRE 7.13. Historical rank of the empirical covariation matrix (5.11) based on time windows of $M=100$ market yields with 33 different times to maturity $\tau_{i} \in\{0.25,0.5,0.75,1,2,3, \ldots, 30\}$. For comparison, the plot also features the average ranks obtained in simulations of the Hull-White extended affine models (V1) and (CIR11) as well as their CRC counterparts (V4) and (CIR 4). These models were calibrated using time windows of $M=100$ observations. The missing values in (CIR/1) and (CIR/4) are due to non-admissible negative levels of mean reversion at these dates, see Section 7.4 and Figure 7.9. The averages are taken over $10^{3}$ simulated paths. In the numerical computation of the rank, eigenvalues which are $10^{-6}$ times smaller than the largest eigenvalue are rounded down to zero.

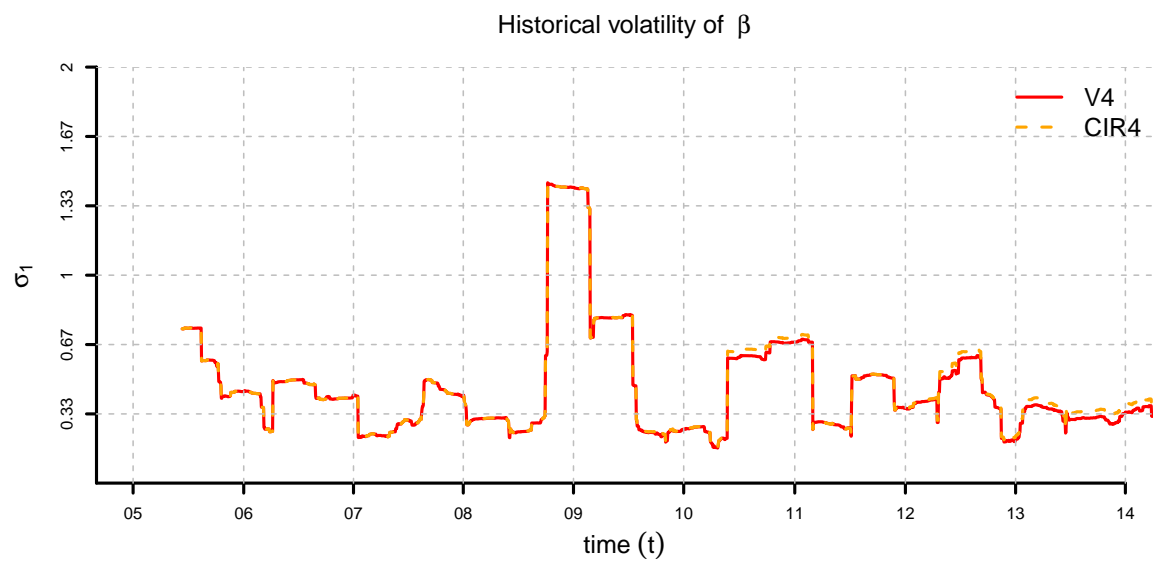

Figure 7.14. Historical values of the parameter $\sigma_{1}$ in the models (V4) and (CIR/4) defined in Section 7.5 estimated using time windows of $M=100$ observations. 


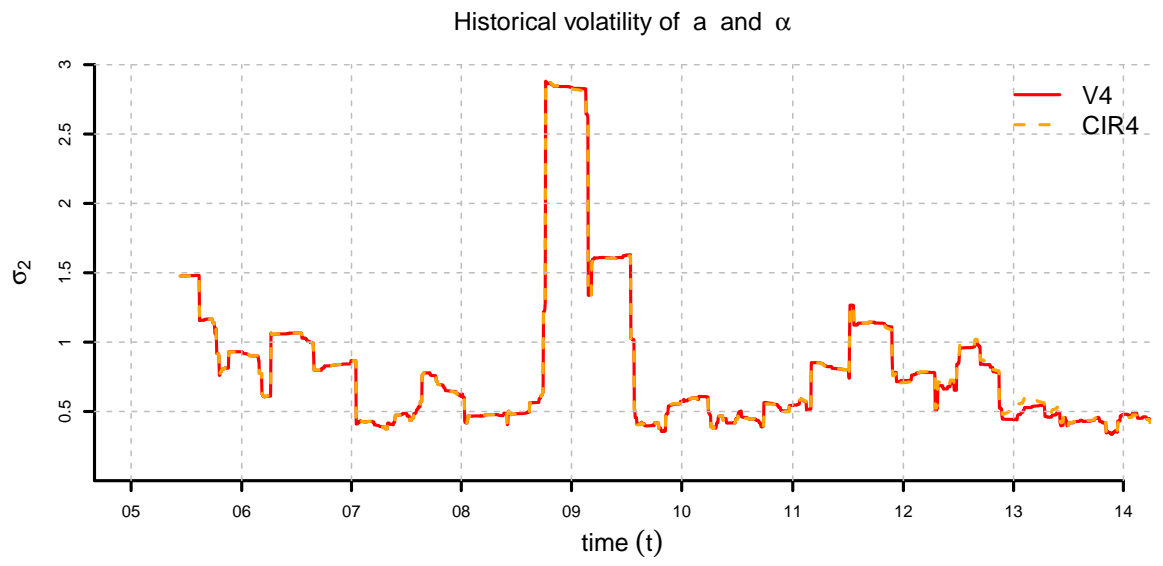

FiguRE 7.15. Historical values of the parameter $\sigma_{2}$ in the models (V4) and (CIR 4 ) defined in Section 7.5 estimated using time windows of $M=100$ observations.

\section{Appendix A. Consistent Recalibration of Cox-Ingersoll-Ross models}

A.1. Overview. We describe CRC models based on CIR short rates, giving a detailed description of the simulation and calibration schemes. For comparison, we briefly digress to the CIR++ model and its CRC version.

A.2. Setup and notation. We use the setup of Sections 3.2 and 4.2 , setting $\mathbb{X}=\mathbb{R}_{+}, \ell=$ $0, \lambda=1$. We do not specify the parameter space $\mathbb{Y}$, yet, but we assume that for each $(x, y) \in$ $\mathbb{X} \times \mathbb{Y}$, the volatility and drift coefficients are given by $A_{y}(x)=\alpha_{y} x$ and $B_{y}(x)=\beta_{y} x$ for some $\alpha_{y} \in(0, \infty)$ and $\beta_{y} \in(-\infty, 0)$. For simplicity, we again choose equidistant grids of times $t_{n}=n \delta$ and times to maturity $\tau_{n}=n \delta$, for all $n \in \mathbb{N}_{0}$, where $\delta$ is a positive constant.

A.3. Hull-White extended Cox-Ingersoll-Ross models. For each fixed set of parameters $(y, \theta) \in \mathbb{Y} \times C\left(\mathbb{R}_{+}\right)$, the $\mathrm{SDE}$ for the short rate process is

$$
d r(t)=\left(\theta(t)+\beta_{y} r(t)\right) d t+\sqrt{\alpha_{y} r(t)} d W(t),
$$

where $W$ is one-dimensional $(\mathcal{F}(t))_{t \geq 0}$-Brownian motion. Thus, $(y, \theta)$ satisfies Assumption 3.1 if and only if $\theta(t) \geq 0$, for all $t \in \mathbb{R}_{+}$. The functional characteristics $(F, R)$ from Section 3.4 are

$$
F_{y}(u)=0, \quad R_{y}(u)=\frac{\alpha_{y}}{2} u^{2}+\beta_{y} u, \quad \text { for all } u \in \mathbb{R} .
$$

Letting $\gamma_{y}=\sqrt{\beta_{y}^{2}+2 \alpha_{y}}$, the solutions of the corresponding Riccati equations are

$$
\Phi_{y}(t)=0, \quad \Psi_{y}(t)=\frac{-2\left(e^{\gamma_{y} t}-1\right)}{\gamma_{y}\left(e^{\gamma_{y} t}+1\right)-\beta_{y}\left(e^{\gamma_{y} t}-1\right)}, \quad \text { for all } t \geq 0 .
$$


Thus, by Theorem 3.3, the forward rates in the Hull-White extended CIR model A.1) with fixed parameters $(y, \theta)$ are given by $h(t)=\mathcal{H}_{y}(\mathcal{S}(t) \theta, r(t))$, where

$$
\mathcal{H}_{y}(\theta, x)(\tau)=-\int_{0}^{\tau} \theta(s) \Psi_{y}^{\prime}(\tau-s) d s-\Psi_{y}^{\prime}(\tau) x, \quad \text { for all }(x, \tau) \in \mathbb{R} \times \mathbb{R}_{+} .
$$

In contrast to the Vasiček model, the integral kernel $\Psi_{y}^{\prime}$ is more complicated,

$$
\Psi_{y}^{\prime}(\tau)=\frac{-2 \gamma_{y} e^{\gamma_{y} \tau}}{\gamma_{y}\left(e^{\gamma_{y} \tau}+1\right)-\beta_{y}\left(e^{\gamma_{y} \tau}-1\right)}+\frac{2\left(e^{\gamma_{y} \tau}-1\right) \gamma_{y} e^{\gamma_{y} \tau}\left(\gamma_{y}-\beta_{y}\right)}{\left(\gamma_{y}\left(e^{\gamma_{y} \tau}+1\right)-\beta_{y}\left(e^{\gamma_{y} \tau}-1\right)\right)^{2}},
$$

and there does not seem to be a closed-form expression for $\theta=\mathcal{C}_{y}(h, x)$. Instead, it must be calculated numerically as described in Section 3.8.

The HJM drift and volatility are

$$
\mu_{y}^{\mathrm{HJM}}(x)(\tau)=\Psi_{y}^{\prime}(\tau) \Psi_{y}(\tau) \alpha_{y} x, \quad \sigma_{y}^{\mathrm{HJM}}(x)(\tau)=-\sqrt{\alpha_{y} x} \Psi_{y}^{\prime}(\tau),
$$

and the HJM equation for forward rates reads as

$$
d h(t)=\left(\mathcal{A} h(t)+\mu_{y}^{\mathrm{HJM}}(h(t)(0))\right) d t+\sigma_{y}^{\mathrm{HJM}}(h(t)(0)) d W(t) .
$$

A.4. Cox-Ingersoll-Ross CRC models. As the factor process is a function of the forward rate process (i.e., $X(t)=r(t)=h(t, 0)$ ), the corresponding CRC models can be characterised by the process $(h, Y)$ instead of $(h, X, Y)$. Thus, in accordance with Theorem 4.5 and Definition 4.6, a process $(h, Y)$ with values in $\mathbb{H} \times \mathbb{Y}$ may be called a CRC model if $h$ satisfies the SPDE

$$
d h(t)=\left(\mathcal{A} h(t)+\mu_{Y(t)}^{\mathrm{HJM}}(h(t)(0))\right) d t+\sigma_{Y(t)}^{\mathrm{HJM}}(h(t)(0)) d W(t),
$$

with drift $\mu_{Y(t)}^{\mathrm{HJM}}$ and volatility $\sigma_{Y(t)}^{\mathrm{HJM}}$ as defined in Equation (A.3). To ensure that the drift and volatility are well-defined, for all $t \in \mathbb{R}_{+}$, it must be assumed that $h(t)(0) \geq 0$ holds, for all $t \in \mathbb{R}_{+}$. The maximally admissible set $\mathcal{I}$ in the CIR model is exactly characterised by this condition.

A.5. Simulation of Cox-Ingersoll-Ross CRC models. The CRC model is simulated as described in Algorithm 4.2. Discretisation in time and time to maturity is done as for the Vasiček model. However, in contrast to the Vasiček model, simulating the short rate process and calibrating Hull-White extensions is done by numerical approximations of order two. The resulting algorithm is presented below.

Algorithm A.1 (Simulation). Given an initial curve of forward rates $h(0)$ and the parameter process $Y$, execute iteratively the following steps, for each $n \in \mathbb{N}_{0}$ :

(i) The values of $\theta\left(t_{n}\right)=\mathcal{C}_{Y\left(t_{n}\right)}\left(h\left(t_{n}\right), r\left(t_{n}\right)\right)$ at times to maturity 0 and $\delta$ are calculated by applying Lemma 3.8 to $g=-h\left(t_{n}\right)-\Psi_{Y\left(t_{n}\right)}^{\prime} r\left(t_{n}\right)$ :

$$
\begin{aligned}
& \theta\left(t_{n}\right)(0)=\mathcal{A} h\left(t_{n}\right)(0)-\beta_{Y\left(t_{n}\right)} h\left(t_{i}\right)(0), \\
& \theta\left(t_{n}\right)(\delta)=\frac{2}{\delta}\left(h\left(t_{n}\right)(\delta)+\Psi_{Y\left(t_{n}\right)}^{\prime}(\delta) r\left(t_{n}\right)\right)+\Psi_{Y\left(t_{n}\right)}^{\prime}(\delta) \theta\left(t_{n}\right)(0) .
\end{aligned}
$$

(ii) A random draw $r\left(t_{n+1}\right)=X_{Y\left(t_{n}\right), \theta\left(t_{n}\right)}^{t_{n}, X\left(t_{n}\right)}\left(t_{n+1}\right)$ of the CIR process with parameter $Y\left(t_{n}\right)$ and time-dependent drift $\theta\left(t_{n}\right)$ is created using the second-order scheme of [1]. 
(iii) $\left(h\left(t_{n+1}\right), \mathcal{A} h\left(t_{n+1}\right)\right)$ is calculated from $\left(h\left(t_{n}\right), \mathcal{A} h\left(t_{n}\right), r\left(t_{n+1}\right)\right)$ using Lemma 4.3 with integrals approximated by the trapezoid rule:

$$
\begin{gathered}
h\left(t_{n+1}\right)(\tau)=h\left(t_{n}\right)(\delta+\tau)+\Psi_{Y\left(t_{n}\right)}^{\prime}(\delta+\tau) r\left(t_{n}\right)-\Psi_{Y\left(t_{n}\right)}^{\prime}(\tau) r\left(t_{n+1}\right) \\
+\frac{\delta}{2}\left(\theta\left(t_{n}\right)(0) \Psi_{Y\left(t_{n}\right)}^{\prime}(\delta+\tau)+\theta\left(t_{n}\right)(\delta) \Psi_{Y\left(t_{n}\right)}^{\prime}(\tau)\right), \\
\mathcal{A} h\left(t_{n+1}\right)(\tau)=\mathcal{A} h\left(t_{n}\right)(\delta+\tau)+\Psi_{Y\left(t_{n}\right)}^{\prime \prime}(\delta+\tau) r\left(t_{n}\right)-\Psi_{Y\left(t_{n}\right)}^{\prime \prime}(\tau) r\left(t_{n+1}\right) \\
+\frac{\delta}{2}\left(\theta\left(t_{n}\right)(0) \Psi_{Y\left(t_{n}\right)}^{\prime \prime}(\delta+\tau)+\theta\left(t_{n}\right)(\delta) \Psi_{Y\left(t_{n}\right)}^{\prime \prime}(\tau)\right) .
\end{gathered}
$$

Here, $h\left(t_{n+1}\right)$ must be calculated at all times to maturity $\tau_{i}$, whereas $\mathcal{A} h\left(t_{n+1}\right)$ is needed only at $\tau_{0}=0$.

A.6. Calibration of Cox-Ingersoll-Ross CRC models. We proceed as in the Vasiček case described in Section 5.9, with Equation (5.11) replaced by

$$
\frac{\left[\widehat{r}\left(\cdot, \tau_{i}\right), \widehat{r}\left(\cdot, \tau_{j}\right)\right]\left(t_{n}\right)-\left[\widehat{r}\left(\cdot, \tau_{i}\right), \widehat{r}\left(\cdot, \tau_{j}\right)\right]\left(t_{n-M}\right)}{\delta \sum_{m=n-M+1}^{n} \widehat{r}\left(t_{m}, \tau_{k}\right)} \approx \alpha \frac{\Psi\left(\tau_{i}\right)}{\tau_{i}} \frac{\Psi\left(\tau_{j}\right)}{\tau_{j}} .
$$

The function $\Psi$ depends on $\alpha, \beta$ as shown in Equation (A.2).

A.7. CIR++ models in the CRC framework. In the CIR++ model 7, Section 3.9], also known as deterministic shift-extended CIR model, the short rate process is defined by $r(t)=X(t)+\theta(t)$, where $X$ is a CIR process and $\theta$ is a deterministic function of time. Note that this is a different time-inhomogeneity than the one described in Section A.3. In particular, the factor process $X$ is time-homogeneous and does not coincide with the short rate.

Forward rate curves are given by

$$
h(t)=\mathcal{S}(t) \theta-b_{y} \Psi_{y}-\Psi_{y}^{\prime} X(t),
$$

where $\Psi_{y}$ is the same as in the CIR case, see Equation (A.2) Given the parameter vector $y$ and the factor $X$, this equation allows to calibrate $\theta$ to a given yield curve without having to invert a Volterra integral operator. The HJM equation of the CIR++ model is

$$
\begin{aligned}
d h(t) & =\left(\mathcal{A} h(t)+\mu_{y}^{\mathrm{HJM}}(X(t))\right) d t+\sigma_{y}^{\mathrm{HJM}}(X(t)) d W(t), \\
d X(t) & =\left(b_{y}+\beta_{y} X(t)\right) d t+\sqrt{\alpha_{y} X(t)} d W(t),
\end{aligned}
$$

where $\mu_{y}^{\mathrm{HJM}}$ and $\sigma_{y}^{\mathrm{HJM}}$ are the same as in the CIR case, see Equation (A.3).

The CRC extension of the CIR++ model is obtained by replacing the constant parameter vector $y$ in (A.7) by a stochastic process $(Y(t))_{t \geq 0}$. The resulting equation is easier to handle than its CIR counterpart for two reasons. First, there are no boundary conditions on $h$. Indeed, $\theta$ is allowed to assume negative values and can be calibrated to any forward rate curve. Thus, Equation (A.7) is defined on the entire space $\mathbb{H} \times \mathbb{R}_{+}$. Second, the SDE for $X$ does not depend on $h$. Therefore, one can first solve for $X$, and then construct a mild solution $h$ by stochastic convolution [13, Section 6.1]:

$$
h(t)=\mathcal{S}(t) h(0)+\int_{0}^{t} \mathcal{S}_{t-s} \mu_{Y(s)}^{\mathrm{HJM}}(X(s)) d s+\int_{0}^{t} \mathcal{S}_{t-s} \sigma_{Y(s)}^{\mathrm{HJM}}(X(s)) d W(s) .
$$

The SDE for $X$ is finite-dimensional. Therefore, existence and uniqueness of $X$ can be shown by standard methods. For example, assuming that $Y$ is independent of $W$, one can condition on $Y$ and use results on time-inhomogeneous affine processes 18 to construct $X$. 
Simulation of the CRC model is analogue to Algorithm 4.2. The recalibration step is easier because no Volterra equation is involved.

A disadvantage of the model is the presence of the hidden factor $X$. In contrast to the CIR version, $X$ is not a function of the forward rate curve and cannot be directly observed. This is a challenge for calibration. We suggest an analogue approach to Section 4.12. First, $\beta_{Y(t)}, \sigma_{Y(t)}$, and $X(t)$ can be identified from the instantaneous covariation

$$
d\left[r\left(\cdot, \tau_{i}\right), r\left(\cdot, \tau_{j}\right)\right](t)=\alpha_{Y(t)} \frac{\Psi_{Y(t)}\left(\tau_{i}\right)}{\tau_{i}} \frac{\Psi_{Y(t)}\left(\tau_{j}\right)}{\tau_{j}} X(t) d t,
$$

of yields with times to maturity $\tau_{i}, \tau_{j}$. Subsequently, $b_{Y(t)}$ can be calibrated by least squares to the prevailing yield curve. Note that in this approach, $X(t)$ is identified from the yield curve dynamics instead of extracted from the prevailing yield curve as in the Vasiček and CIR cases. For this reason the calibration is expected to be numerically more difficult.

\section{REFERENCES}

[1] A. Alfonsi. "High order discretization schemes for the CIR process: Application to affine term structure and Heston models". In: Mathematics of Computation 79.269 (2010), pp. 209-237.

[2] W. Arendt, C. J. Batty, M. Hieber, and F. Neubrander. Vector-valued Laplace transforms and Cauchy problems. Vol. 96. Springer Science \&amp; Business Media, 2011.

[3] B. for International Settlements (BIS). Basel Committee on Banking Supervision (BCBS). Basel II: International convergence of capital measurement and capital standards. A revised framework-Comprehensive version. 2006. URL: http://www bis.org/publ/bcbs128.htm (visited on 05/2016).

[4] G. Barone-Adesi, F. Bourgoin, and K. Giannopoulos. "Market Risk: Don't Look Back". In: Risk 11 (Aug. 1998), pp. 100-103.

[5] F. Baudoin, J. Teichmann, et al. "Hypoellipticity in infinite dimensions and an application in interest rate theory". In: The Annals of Applied Probability 15.3 (2005), pp. 1765-1777.

[6] J.-P. Bouchaud et al. "Phenomenology of the interest rate curve". In: Applied Mathematical Finance 6.3 (1999), pp. 209-232.

[7] D. Brigo and F. Mercurio. Interest Rate Models - Theory and Practice. Springer, 2007.

[8] H. Brunner. Collocation methods for Volterra integral and related functional differential equations. Vol. 15. Cambridge University Press, 2004.

[9] R. Carmona and S. Nadtochiy. "Tangent Lévy market models". In: Finance Stoch. 16.1 (2012), pp. 63-104.

[10] C. Chiarella, D. Colwell, and O. K. Kwon. "A Class of Stochastic Volatility HJM Interest Rate Models". In: Inaugural Derivatives Research Workshop, Melbourne Derivatives Research Group, University of Melbourne. 2004.

[11] R. Cont. "Modeling term structure dynamics: an infinite dimensional approach". In: International Journal of Theoretical and Applied Finance 8.03 (2005), pp. 357-380.

[12] C. Cuchiero and J. Teichmann. "Fourier transform methods for pathwise covariance estimation in the presence of jumps". In: arXiv:1301.3602 (2013).

[13] G. Da Prato and J. Zabczyk. Stochastic Equations in Infinite Dimension. Cambridge University Press, 2014. 
[14] P. Dörsek and J. Teichmann. "Efficient simulation and calibration of general HJM models by splitting schemes". In: SIAM Journal on Financial Mathematics 4.1 (2013), pp. 575-598.

[15] D. Duffie, D. Filipović, and W. Schachermayer. "Affine processes and applications in finance". In: Ann. Appl. Probab. 13.3 (2003), pp. 984-1053.

[16] D. Filipović. Consistency problems for Heath-Jarrow-Morton interest rate models. Springer, 2001.

[17] D. Filipović. Term-structure models. Springer Finance. A graduate course. Berlin: Springer-Verlag, 2009.

[18] D. Filipović. "Time-inhomogeneous affine processes". In: Stochastic Process. Appl. 115.4 (2005), pp. 639-659.

[19] A. Gombani, S. R. Jaschke, and W. J. Runggaldier. "A filtered no arbitrage model for term structures from noisy data". In: Stochastic processes and their applications 115.3 (2005), pp. 381-400.

[20] E. Hansen and A. Ostermann. "Exponential splitting for unbounded operators". In: Mathematics of Computation 78.267 (2009), pp. 1485-1496.

[21] P. Harms, D. Stefanovits, and J. Teichmann. Exponential moments of time-inhomogeneous affine processes. Work in progress.

[22] P. Harms, D. Stefanovits, J. Teichmann, and M. V. Wüthrich. "Consistent recalibration of the discrete-time multi-factor Vasiček model". In: Risks 4.3 (2016), pp. 131.

[23] M. Henseler, C. Peters, and R. C. Seydel. "A Tractable Multi-Factor Dynamic Term-Structure Model for Risk Management". In: SSRN 2225738 (2013).

[24] J. Kallsen and P. Krühner. "On a Heath-Jarrow-Morton approach for stock options". In: arXiv:1305.5621 (2013).

[25] P. Linz. "Numerical methods for Volterra integral equations of the first kind." In: Comput. J. 12 (1969), pp. 393-397.

[26] R. B. Litterman and J. Scheinkman. "Common factors affecting bond returns". In: The Journal of Fixed Income 1.1 (1991), pp. 54-61.

[27] T. Nakayama. "Support Theorem for Mild Solutions of SDE's in Hilbert Spaces". In: Journal of Mathematical Sciences 11.3 (2004), pp. 245-312.

[28] J.-P. Ortega, R. Pullirsch, J. Teichmann, and J. Wergieluk. "A new approach for scenario generation in Risk management". In: arXiv:0904.0624 (2009).

[29] A. Richter and J. Teichmann. "Discrete time term structure theory and consistent recalibration models". In: arXiv:1409.1830 (2014).

[30] L. C. G. Rogers and D. Williams. Diffusions, Markov processes and martingales: Volume 2, Itō calculus. Vol. 2. Cambridge university press, 2000.

[31] L. E. Svensson. Estimating and interpreting forward interest rates: Sweden 1992-1994. Tech. rep. National Bureau of Economic Research, 1994.

[32] J. Teichmann and M. V. Wüthrich. "Consistent long-term yield curve prediction". In: arXiv:1203.2017 (2012).

[33] The new euro area yield curves. Tech. rep. European Central Bank Monthly Bulletin, Feb. 2008. 
Institute of Mathematics, Albert-Ludwigs University Freiburg

E-mail address: philipp.harms@stochastik.uni-freiburg.de

Department of Mathematics, ETH Zürich

E-mail address: david.stefanovits@math.ethz.ch

E-mail address: josef.teichmann@math.ethz.ch

E-mail address: mario.wuethrich@math.ethz.ch 